René Spencer Chatwell, Matthias Heinen, Jadran Vrabec

\title{
Diffusion limited evaporation of a binary liquid film
}

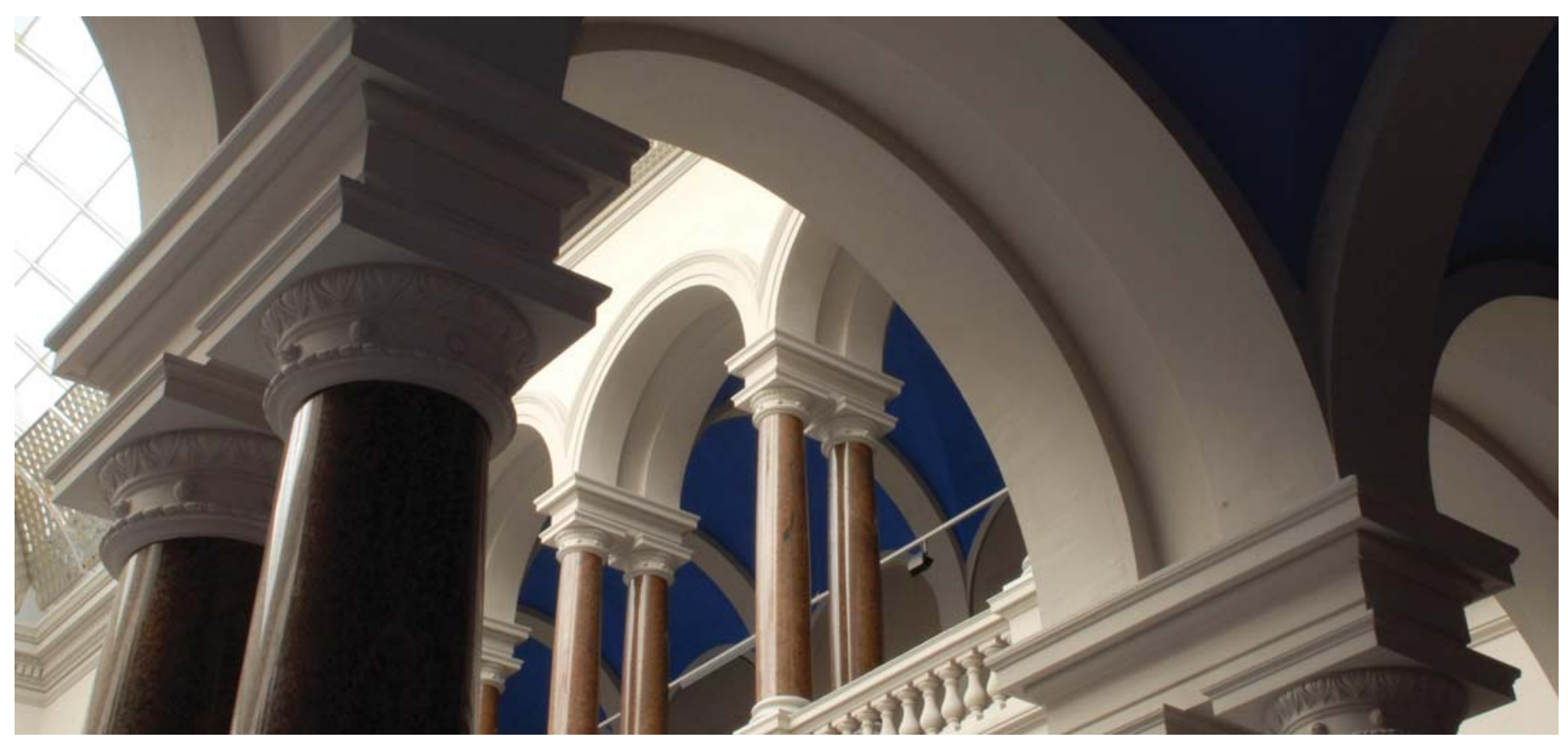

Chatwell, René Spencer; Heinen, Matthias; Vrabec, Jadran (2019). Diffusion limited evaporation of a binary liquid film. International J ournal of Heat and Mass Transfer, 132, 1296-1305.

https://doi.org/10.1016/j.ijheatmasstransfer.2018.12.030 


\title{
Diffusion limited evaporation of a binary liquid film
}

\author{
René Spencer Chatwell ${ }^{\mathrm{a}}$, Matthias Heinen ${ }^{\mathrm{a}}$, Jadran Vrabec ${ }^{\mathrm{a}, *}$ \\ ${ }^{a}$ Thermodynamics and Process Engineering, Technical University of Berlin, 10587 Berlin, \\ Germany
}

\begin{abstract}
An analytical solution of a model fluid's time behavior, known as the Stefan problem, is presented. A scenario is investigated in which a planar twocomponent liquid film is continuously evaporating into a thermodynamically non-ideal vapor phase. Evaporation is initiated and maintained by a spatial chemical potential gradient, while its rate is limited by the components' diffusion fluxes across the vapor-liquid interface. Local thermodynamic equilibrium is found to be present throughout the process. In contrast to the classical approach relying on equations of state, all required non-idealities are formulated in relation to the Gibbs energy and are determined by molecular simulations. Initially, the liquid is an equimolar mixture of two components of different volatility, whereas the adjacent vapor phase is dominated by a dense inert gas. To validate the analytical model and verify all exploited assumptions, the results are contrasted to large scale molecular dynamics simulations.
\end{abstract}

Keywords: evaporative mass transfer, analytical modeling, large scale molecular dynamics simulations

\section{Introduction}

Investigations of phase transitions for spherical and planar geometries are methodically similar. Historically, research was focused on droplet evaporation indicating a relationship between the current square diameter $d^{2}(t)$ and an initial

*vrabec@tu-berlin.de

This is the Accepted Manuscript of: Chatwell, R. S., Heinen, M., \& Vrabec, J. (2019). Diffusion limited evaporation of a binary liquid film. International Journal of Heat and Mass Transfer, 132, 1296-1305. https://doi.org/10.1016/j.ijheatmasstransfer.2018.12.030

This work is licensed under a Creative Commons Attribution-NonCommercial-NoDerivatives 4.0 International License, http://creativecommons.org/licenses/by-nc-nd/4.0/. 
value $d_{0}^{2}$ to exist, which for pure liquids is linear in time $t$

$$
d^{2}(t)=d_{0}^{2}-K t
$$

For droplets immersed into a quiescent atmosphere, the evaporation rate $K$ is exclusively a function of the thermodynamic state. The literature refers to this equation as "d-squared law" [1. The problem's more thorough theoretical treatments originated from around the early twentieth century [2, 3], whereas a first estimation of $K$ had already been conducted by Maxwell [4] in his 1877 work on "Diffusion" and was presented in its current form by Fuchs [ 5

$$
K=\frac{8 D \rho_{v}}{\rho_{l}}\left(y_{e, s}-y_{e, \infty}\right),
$$

where $y_{e, i}$ represent the mole fraction of evaporate at either surface or in sufficient distance from the droplet. This mole fraction difference initiates and maintains the evaporation process while a diffusion coefficient $D$ in combination with the vapor-liquid density ratio $\rho_{v} \rho_{l}^{-1}$ determines its rate. Maxwell's approach was later refined, among others by Spalding [6], to account for substantial evaporation rates that are indicative of an evaporate's high vapor pressure and results in its accumulation in the interface region between liquid and vapor. Consequently, a more detailed description of the droplet's surface composition became indispensable and necessitated Spalding to take the quiescent atmospheric gas' mole fraction $y_{a, s}$ into account

$$
K=\frac{8 D \rho_{v}}{\rho_{l}} \ln \left(1+\frac{y_{e, s}-y_{e, \infty}}{y_{a, s}}\right) .
$$

In fact, Maxwell's description (2) represents the first-order approximation of Spalding's formulation (3) for the case of a dominating atmospheric gas at droplet surface, i.e. $y_{a, s} \approx 1$. Maxwell and Spalding assumed both bulk phases

5 to be constantly in vapor-liquid equilibrium that due to mass transport is only sustainable via continuous evaporation.

The d-squared law in its form (1) has been experimentally validated over a substantial range of thermodynamic states as well as for a variety of nonsooting monocomponent droplets through a wider spectrum of chemical com1o plexity [7, 8, 9, 10, 11, 12, 13, 14, 15, 16. Multicomponent droplets, however, 
offer a conspicuously different behavior owed to the continuous variation in molar composition of both liquid $\boldsymbol{x}(t)$ and coexisting vapor $\boldsymbol{y}(t)$. Species with genuinely dissimilar properties lead to evaporation rates that vary substantially over time [17, 18, 19]. Complementing theoretical and experimental work, mul-

15 ticomponent evaporation has also been successfully addressed by atomistic simulations [20, 21].

\section{Object under study}

Complementary to the literature's focus on spherical droplets, this work solves the Stefan problem [22, 23, for a multicomponent liquid film analytically.

20 This analytical solution, representing the d-squared law's (1) analogue for planar surfaces, is then validated by large scale molecular dynamics (MD) simulations. Consequently, a two-component liquid film's change in molar composition $\boldsymbol{x}(t)$ and thickness $\xi(t)$ is investigated during evaporation into an inert gas dominated vapor phase. Each phase is composed of components with deliberately specified

25 characteristics, cf. appendix A. Due to these characteristics, in combination with the selected thermodynamic state, outlined in table 1 both bulk phases are thermodynamically non-ideal. An adequate description of the establishing evaporation dynamics requires all component's diffusion coefficients, liquid phase activity coefficients and vapor phase fugacities to be known as functions sо of the present state.

Molecular simulations are employed for two different reasons. For one, the sampling of all required thermodynamic observables by dedicated MD and Monte Carlo (MC) simulations requires substantially less approximation than modeling resting on equations of state. On the other hand, the present hydrodynamic formulation addresses the phases' molar composition $\boldsymbol{x}(t), \boldsymbol{y}(t)$ directly. Large scale MD simulations offer an alternative access to determine a phase's molar composition with a spatial and temporal resolution that is orders of magnitude greater than what is currently achievable by experiment. To be comparable to hydrodynamic length and time scales, both a sufficiently large 


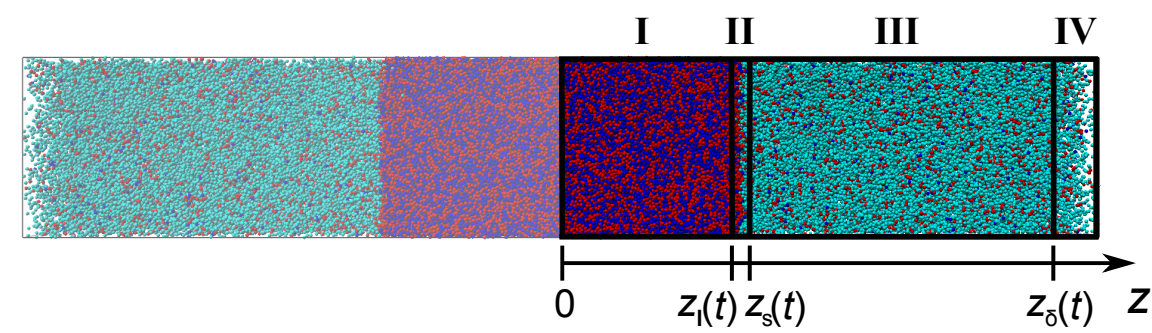

Figure 1: Snapshot taken from one of the present large scale MD simulations depicting the scenario in vapor-liquid equilibrium, i.e. prior to evaporation. The studied domain is decomposed into four regions that are connected physically on microscopic length scales. The central role is attributable to the interphase (II), characterized by a steep density decline ranging from liquid (I) to vapor (III). A chemical potential gradient is established by substituting evaporated particles that originated from the liquid phase by inert gas particles within the control volume (IV). The scenario's symmetry ensures the net molar flux to be zero at the origin $z=0$, while selective thermostatisation establishes a spatially and temporally constant temperature $T=80 \mathrm{~K}$ which is accompanied by a pressure of $p=6.34 \mathrm{MPa}$ for the present mixture.

ensemble and a substantial simulated time is necessary. Figure 1 outlines an atomistic representation of the investigated scenario containing $2.5 \cdot 10^{5}$ particles with an initial liquid film and vapor phase thickness of $z_{l}\left(t_{0}\right)=15 \mathrm{~nm}$ and $\delta=z_{\delta}(t)-z_{s}(t)=30 \mathrm{~nm}$, respectively. Various separate simulation series were carried out, to either sample thermodynamic observables or to pursue large scale

45 MD simulations, cf. Appendix A.

The present hydrodynamic formulation rests on a fluid domain decomposition into four separate regions, as outlined in figure 1. The liquid phase (I) initially consists of two equimolarly mixed components (1 and 2) with different volatility, that in this context should be understood as an attribute for the comso ponent's endeavor to transition from the liquid and to remain in the coexisting vapor phase (III). The latter was chosen to be dominated by an inert gas (component 3 ), which was specified not to be condensable and be barely miscible in the liquid film. Both bulk phases are physically coupled by an interface region (II) that is addressed as interphase in the following. A control volume (IV) 55 representing the invariant atmospheric conditions completes the fluid domain. 


\subsection{Local thermodynamic equilibrium}

Under vapor-liquid equilibrium the particles' entire motion is thermal and consequently drift-free Maxwellian. The applied chemical potential gradient initiates evaporation dynamics by introducing a collective drift, i.e hydrodynamic contribution $\boldsymbol{u}_{i}$, to the $i$-th component's particle velocity $\boldsymbol{v}_{i}$, that superimposes to the random thermal motion $\boldsymbol{w}_{i}$

$$
\boldsymbol{v}_{i}=\boldsymbol{u}_{i}+\boldsymbol{w}_{i}
$$

Local thermal equilibrium exists in every spatial domain (I) - (IV) if all particles' thermal velocities are Maxwellian distributed. The corresponding velocity distribution function $f_{z, i}$ for the relevant mass transport direction $z$ is most sensibly displayed in its contracted form 24,25 ,

$$
f_{z, i}=\sqrt{\frac{m_{i}}{2 \pi k_{b} T}} \exp \left[-\frac{m_{i}\left(v_{z}-u_{z}\right)^{2}}{2 k_{B} T}\right] .
$$

Particle interactions identify as mechanism that drive a system towards local equilibrium [26]. A dense vapor phase is indicative of such dominating interactions, realized in the present scenario by a high system pressure $p=6.34 \mathrm{MPa}$ ensuring the particles' thermal velocities to be Maxwellian distributed naturally in each domain, even the narrow interphase. To confirm the anticipated Maxwellian distribution (5), additional stationary large scale MD simulations for the liquid film being prepared and maintained at $\boldsymbol{x}^{\dagger}=(0.48,0.52) \mathrm{mol} \mathrm{mol}^{-1}$ were carried out. The results are presented in figure (2) and fully confirm local

65 equilibrium to exist. Similar results were reported in the literature on interfacial mass transfer, for both stationary [27, 28] and instationary [29, 30] cases. Non-Maxwellian distributed particle velocities, in contrast, have conclusively been demonstrated to establish during evaporation into rarefied gas phases or vacuum [31, 32, 33.

\section{3. Hydrodynamic description}

The present formalism models the vapor phase as a classical boundary layer and predicts the film's evaporation rate, i.e. its regression, as well as the change 


\begin{tabular}{cccccccc}
\hline$i$ & $\begin{array}{c}y_{i} / \\
\mathrm{mol} \mathrm{mol}^{-1}\end{array}$ & $\begin{array}{c}\rho_{v, i} / \\
\mathrm{mol} \mathrm{dm}^{-3}\end{array}$ & $\begin{array}{c}\vartheta_{i} / \\
-\end{array}$ & $\begin{array}{c}x_{i} / \\
\mathrm{mol} \mathrm{mol}^{-1}\end{array}$ & $\begin{array}{c}\rho_{0, i} / \\
\mathrm{mol} \mathrm{dm}^{-3}\end{array}$ & $\begin{array}{c}\gamma_{i} / \\
-\end{array}$ & $\begin{array}{c}\phi_{i} / \\
\mathrm{mol} \mathrm{cm}^{-2} \mathrm{~s}^{-1}\end{array}$ \\
\hline 1 & 0.13 & 1.10 & 4.09 & 0.5 & 47.77 & 1.07 & 1.41 \\
2 & 0.03 & 0.23 & 20.07 & 0.5 & 42.33 & 1.06 & 0.13 \\
3 & 0.84 & 7.12 & - & 0 & 8.33 & - & - \\
\hline
\end{tabular}

Table 1: The system was initially prepared in a thermodynamic state with temperature $T=80$ $\mathrm{K}$ and pressure $p=6.34 \mathrm{MPa}$, where both phases are under vapor-liquid equilibrium (VLE) with the given liquid $x_{i}$ and vapor phase mole fractions $y_{i}$ as well as partial vapor $\rho_{v, i}$ and pure component molar densities $\rho_{0, i}$. The thermodynamic non-idealities, i.e. the fugacity coefficient's excess contribution $\vartheta_{i}$, the activity coefficient $\gamma_{i}$ and consequently the diffusion flux's entire thermodynamic contribution $\varphi_{i}$, albeit functions of composition, were considered constant and evaluated under these VLE conditions.

of both phases' molar composition over time, while resting exclusively on particle conservation formulated in integral form.

A domain's change in mass is determined by an imbalance of particle fluxes $j_{z, i}$ through its boundary [34] and yields for the bulk liquid's domain (I) $z \in\left[0, z_{l}(t)\right]$, while invoking the inert gas characteristics not to be condensable and be barely miscible in this liquid

$$
d_{t}\left(\int_{V_{t}} d V\left[\begin{array}{c}
\rho_{l, 1}(z, t) \\
\rho_{l, 2}(z, t)
\end{array}\right]\right)+\oint_{\partial V_{t}} d A\left[\begin{array}{c}
j_{z, 1}(z, t) \\
j_{z, 2}(z, t)
\end{array}\right]=\left[\begin{array}{l}
0 \\
0
\end{array}\right] .
$$

A component's particle density decomposes into mole fraction $x_{i}$ and overall molar density $\rho_{l}$ of the respective phase $\rho_{l, i}=x_{i} \rho_{l}$, whereas the particle flux factorizes to $j_{z, i}=x_{i} \rho_{l} u_{z, i}$, with the convective molar averaged velocity $u_{z, i}$ towards the interphase. Assuming spatial homogeneity of the liquid film's molar composition $x_{i} \neq x_{i}(z)$ and density $\rho_{l} \neq \rho_{l}(z)$ with the additional observation that the particle fluxes are invariant across the domain's surface leads to a straightforward integration, where $V(t)=z_{l}(t) A_{0}$

$$
d_{t}\left[\begin{array}{l}
x_{1}(t) \rho_{l}(t) z_{l}(t) \\
x_{2}(t) \rho_{l}(t) z_{l}(t)
\end{array}\right]+\rho_{l}(t)\left[\begin{array}{l}
x_{1}(t) u_{z, 1}(z, t) \\
x_{2}(t) u_{z, 2}(z, t)
\end{array}\right]=\left[\begin{array}{l}
0 \\
0
\end{array}\right] .
$$




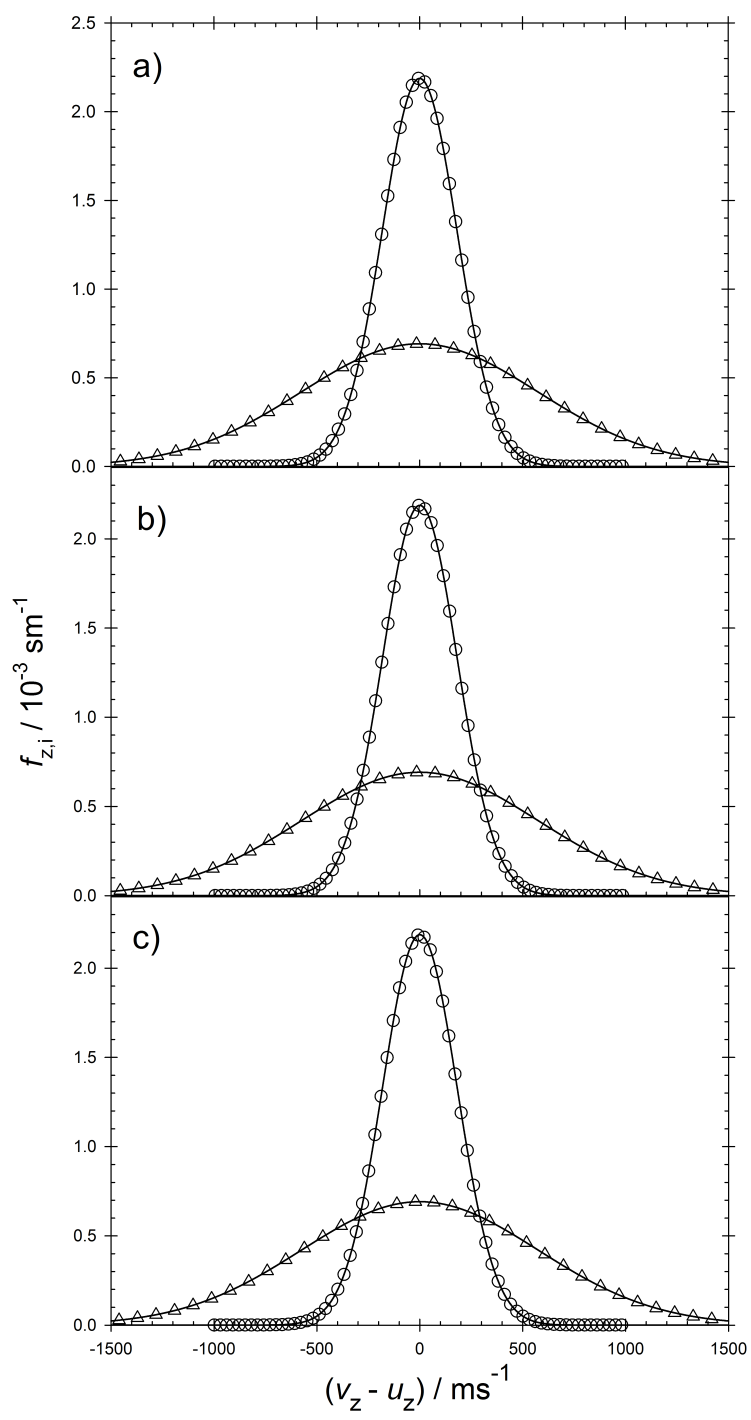

Figure 2: Contrasting the results between drifting Maxwell distributed (solid line), as defined in (5), and the sampled MD velocities for the relevant volatile (triangles) and less-volatile (circles) components does not disclose any disparities. These data were evaluated at temperature $T=80 \mathrm{~K}$ and three different positions ranging from bulk liquid to bulk vapor. The large difference between both component's distribution functions is due to their different molar mass. a) Values sampled in the bulk liquid phase at position $z \leq z_{l}(t)$ in close vicinity to the interphase. b) Values sampled in the interphase at position $z \in] z_{l}(t), z_{s}(t)[$. c) Values sampled in the bulk vapor phase at position $z \geq z_{s}(t)$ in vicinity to the interphase. 
The interphase $z \in] z_{l}(t), z_{s}(t)$ [ balances the molar particle fluxes between both bulk phases, where $j_{z, i}=x_{i}(t) u_{z, i}\left(z_{l}, t\right)$ indicates the $i$-th component's molar flux exiting the bulk liquid phase and $j_{z, i}^{s}=y_{i}\left(z_{s}, t\right) u_{z, i}\left(z_{s}, t\right)$ indicating the flux entering the adjacent bulk vapor phase

$$
-\rho_{l}(t)\left[\begin{array}{l}
x_{1}(t) u_{z, 1}\left(z_{l}, t\right) \\
x_{2}(t) u_{z, 2}\left(z_{l}, t\right)
\end{array}\right]+\rho_{v}\left[\begin{array}{l}
y_{1}\left(z_{s}, t\right) u_{z, 1}\left(z_{s}, t\right) \\
y_{2}\left(z_{s}, t\right) u_{z, 2}\left(z_{s}, t\right)
\end{array}\right]=\left[\begin{array}{l}
0 \\
0
\end{array}\right] .
$$

It is by bringing together (7) and (8) that the connection between the liquid phase's mass and the fluxes in the vapor phase (III) $z \in\left[z_{s}(t), z_{\delta}(t)\right]$ is achieved

$$
d_{t}\left[\begin{array}{l}
x_{1}(t) \rho_{l}(t) z_{l}(t) \\
x_{2}(t) \rho_{l}(t) z_{l}(t)
\end{array}\right]+\rho_{l}(t)\left[\begin{array}{l}
y_{1}\left(z_{s}, t\right) u_{z, 1}\left(z_{s}, t\right) \\
y_{2}\left(z_{s}, t\right) u_{z, 2}\left(z_{s}, t\right)
\end{array}\right]=\left[\begin{array}{l}
0 \\
0
\end{array}\right] .
$$

Although the inert gas is assumed not to be soluble in the liquid, its presence in the interphase and vapor has to be accounted for. Each component's collective drifting motion $y_{i} \boldsymbol{u}_{i}$, as defined in (4), is decomposed further into an advective $y_{i} \boldsymbol{u}$ and a diffusive contribution [35] $y_{i} \boldsymbol{U}_{i}$

$$
y_{i} \boldsymbol{u}_{i}=y_{i}\left(\boldsymbol{u}+\boldsymbol{U}_{i}\right) \text {. }
$$

The evaporate's total convective flux is related to its total diffusive flux, cf. supplementary material, with all velocities being formulated in the mean molar reference frame

$$
\sum_{i=1}^{2} y_{i} u_{z, i}=\frac{y_{1}}{y_{3}} U_{z, 1}+\frac{y_{2}}{y_{3}} U_{z, 2}
$$

and it is this correlation that renders the evaporation process diffusion driven. It is not necessarily trivial to assume the vapor phase mole fraction quotients to be time invariant

$$
b_{i 3}(z)=\frac{y_{i}(z, t)}{y_{3}(z, t)}
$$

yet this simplification still allows to reasonably predict the vapor phase's molar composition. To ease the notation $\mathcal{L}_{z, i}=b_{i 3} U_{z, i}$ is set for each component's diffusive flux. 


\subsection{Diffusive motion}

A general description of diffusive motion in an inhomogeneous medium is given by the Fokker-Planck diffusivity law [36]. In the present case, this flux has to be computed adjacent to the interphase, i.e at $z=z_{s}(t)$

$$
\left[\begin{array}{c}
\mathcal{L}_{z, 1}\left(z_{s}\right) \\
\mathcal{L}_{z, 2}\left(z_{s}\right)
\end{array}\right]=-\left.\partial_{z}\left(\left[\begin{array}{cc}
D_{11}(\boldsymbol{y}) & D_{12}(\boldsymbol{y}) \\
D_{21}(\boldsymbol{y}) & D_{22}(\boldsymbol{y})
\end{array}\right] \cdot\left[\begin{array}{c}
b_{13}(z) \\
b_{23}(z)
\end{array}\right]\right)\right|_{z_{s}},
$$

wherein the Fick diffusion coefficients $D_{i j}$ express the phenomenologically postulated proportionality between flux and respective mole fraction gradient [37. For multicomponent systems, however, each diffusive flux is the result of all driving gradients. The diffusion matrix, containing Fick's coefficients $D_{i j}$, is separable into a kinetic and a thermodynamic contribution [38]

$$
\left[\begin{array}{ll}
D_{11} & D_{12} \\
D_{21} & D_{22}
\end{array}\right]=\left[\begin{array}{ll}
B_{11} & B_{12} \\
B_{21} & B_{22}
\end{array}\right]^{-1} \cdot\left[\begin{array}{cc}
\Gamma_{11} & \Gamma_{12} \\
\Gamma_{21} & \Gamma_{22}
\end{array}\right],
$$

75 the former being the Maxwell-Stefan and the latter the thermodynamic factor matrix, describing a mixture's departure from ideality and being composition derivatives of the excess Gibbs energy [39] $g^{e}$. In this work, both matrices were considered constant throughout the entire evaporation process. Assuming the kinetic part to be invariant is justifiable, as for the thermodynamic part, depending strongly on composition, this simplification was confirmed by the performed MD simulations, cf. supplementary material. Bringing together (9), (11), (13) and (14) leads to

$$
\begin{aligned}
d_{t} & {\left[\begin{array}{l}
x_{1}(t) \rho_{l}(t) z_{l}(t) \\
x_{2}(t) \rho_{l}(t) z_{l}(t)
\end{array}\right]-\rho_{v}\left[\begin{array}{ll}
B_{11} & B_{12} \\
B_{21} & B_{22}
\end{array}\right]^{-1} \cdot\left[\begin{array}{cc}
\Gamma_{11} & \Gamma_{12} \\
\Gamma_{21} & \Gamma_{22}
\end{array}\right] } \\
\times & \left.d_{z}\left[\begin{array}{l}
b_{13}(z) \\
b_{23}(z)
\end{array}\right]\right|_{z_{s}}=\left[\begin{array}{l}
0 \\
0
\end{array}\right] .
\end{aligned}
$$

The diffusion matrix couples both fluxes, its coupling strength is measured by how much the eigenvector matrix $\mathbf{T}$ differs from the identity matrix $\mathbf{I}$, and can 
spectrally be decomposed

$$
\left[\begin{array}{ll}
B_{11} & B_{12} \\
B_{21} & B_{22}
\end{array}\right]^{-1} \cdot\left[\begin{array}{ll}
\Gamma_{11} & \Gamma_{12} \\
\Gamma_{21} & \Gamma_{22}
\end{array}\right]=\mathbf{T} \cdot\left[\begin{array}{cc}
\mathcal{D}_{1} & 0 \\
0 & \mathcal{D}_{2}
\end{array}\right] \cdot \mathbf{T}^{-1} .
$$

The eigenvalues $\mathcal{D}_{i}$ have to be understood as effective diffusion coefficients that map the weighted action of both driving forces onto each flux

$$
\begin{aligned}
\mathbf{T}^{-1} \cdot d_{t} & {\left[\begin{array}{l}
x_{1}(t) \rho_{l}(t) z_{l}(t) \\
x_{2}(t) \rho_{l}(t) z_{l}(t)
\end{array}\right]-\rho_{v}\left[\begin{array}{cc}
\mathcal{D}_{1} & 0 \\
0 & \mathcal{D}_{2}
\end{array}\right] \cdot \mathbf{T}^{-1} } \\
\times & \left.d_{z}\left[\begin{array}{l}
b_{13}(z) \\
b_{23}(z)
\end{array}\right]\right|_{z_{s}}=\left[\begin{array}{l}
0 \\
0
\end{array}\right] .
\end{aligned}
$$

${ }_{85}$ In the attempt to pursue first order effects, the appearing spatial gradient $d_{z}\left(b_{i 3}\right)$ is linearized. The vapor domain $\delta=z_{\delta}(t)-z_{s}(t)$ constitutes a classical boundary layer problem with exemplary boundary conditions out of which the following curvature-gradient correlation $(18)$ is a statement of particle conservation, cf. supplementary material

$$
\begin{aligned}
& z=z_{s}(t): b_{i 3}\left(z_{s}\right)=b_{i 3, s},\left.\quad d_{z z}\left(b_{i 3}\right)\right|_{z_{s}}=\left(\left.d_{z}\left(b_{i 3}\right)\right|_{z_{s}}\right)^{2} \\
& z=z_{\delta}(t): b_{i 3}\left(z_{\delta}\right)=b_{i 3, \infty},\left.\quad d_{z}\left(b_{i 3}\right)\right|_{z_{\infty}}=0 .
\end{aligned}
$$

It has already been established that Fick diffusion originates from a spatial mole fraction gradient comprising the stagnant gas presence, which historically arisen has been termed mass transfer number [6]

$$
B_{i 3}=b_{i 3, \infty}-b_{i 3, s}=\frac{y_{i, \infty}-y_{i, s}}{y_{3, s}} .
$$

9o The nonlinearity in the proposed boundary conditions $(18)$ necessitates a different ansatz to approximate the differential operator by a difference quotient. The simplest function that complies with this set of boundary conditions is a third order polynomial, first introduced by Pohlhausen [40] and later applied to mass transfer problems by Spalding [41]

$$
\begin{aligned}
\frac{b_{i 3}(z)-b_{i 3, s}}{B_{i 3}} & =\alpha_{i 3}\left(\frac{z-z_{s}(t)}{\delta}\right)+\beta_{i 3}\left(\frac{z-z_{s}(t)}{\delta}\right)^{2} \\
& +\gamma_{i 3}\left(\frac{z-z_{s}(t)}{\delta}\right)^{3} .
\end{aligned}
$$




$$
\begin{aligned}
1 & =\alpha_{i 3}+\beta_{i 3}+\gamma_{i 3}, \\
0 & =\alpha_{i 3}^{2}-\frac{2}{B_{i 3}} \beta_{i 3}, \\
0 & =\alpha_{i 3}+2 \beta_{i 3}+3 \gamma_{i 3},
\end{aligned}
$$

with two sets of solutions out of which only the first yields physically sensible boundary layer profiles

$$
\begin{aligned}
\alpha_{i 3} & =\frac{2}{B_{i 3}}\left( \pm \sqrt{1+\frac{3}{2} B_{i 3}}-1\right), \\
\beta_{i 3} & =3+\frac{4}{B_{i 3}}\left(\mp \sqrt{1+\frac{3}{2} B_{i 3}}+1\right), \\
\gamma_{i 3} & =\frac{2}{B_{i 3}}\left( \pm \sqrt{1+\frac{3}{2} B_{i 3}}-1\right)-2 .
\end{aligned}
$$

The binomial theorem allows to approximate square roots to a convenient degree of accuracy, given the fact the transfer number is small $B_{i 3} \ll 1$, and the anticipated linearization is attained by truncating the series approximation to first order

$$
\sqrt{1+\frac{3}{2} B_{i 3}} \approx 1+\frac{3}{4} B_{i 3}
$$

It is by considering this approximation in the coefficients $\alpha_{i 3}, \beta_{i 3}, \gamma_{i 3}$ that 20 exposes the proper derivative's substitution by a difference quotient

$$
\left.d_{z}\left(b_{i 3}\right)\right|_{z_{s}} \approx \alpha_{i 3} \frac{b_{i 3, \infty}-b_{i 3, s}}{\delta} \approx \frac{2}{3 \delta} \frac{y_{i, \infty}-y_{i, s}}{y_{3, s}} .
$$

The proportionality coefficient $\alpha_{i 3}$ in front of the otherwise simple difference quotient is being mandated by the physically required boundary conditions. The present scenario was set up such that an inert gas component dominates the vapor phase, permitting to set $y_{3, s} \approx 1$ even at the surface. Moreover, the composition of the surrounding atmosphere, represented by the control volume, was specified to be $y_{i, \infty}=0$. Inserting the attained linearization into (17), while assuming weak flux coupling $\mathbf{T} \approx \mathbf{I}$, leads to

$$
d_{t}\left[\begin{array}{c}
x_{1}(t) \rho_{l}(t) z_{l}(t) \\
x_{2}(t) \rho_{l}(t) z_{l}(t)
\end{array}\right]+\frac{3 \rho_{v}}{2 \delta}\left[\begin{array}{cc}
\mathcal{D}_{1} & 0 \\
0 & \mathcal{D}_{2}
\end{array}\right] \cdot\left[\begin{array}{c}
y_{1}\left(z_{s}, t\right) \\
y_{2}\left(z_{s}, t\right)
\end{array}\right] \approx\left[\begin{array}{l}
0 \\
0
\end{array}\right]
$$


During local equilibrium, liquid and vapor phase mole fractions $x_{i}, y_{i}$ are entangled and as such cannot be determined independently.

\subsection{Thermodynamic non-ideality}

The central element when formulating phase equilibria is the extensive Gibbs energy $G$ that is constructed by the weighted arithmetic mean out of every component's chemical potential $\mu_{i}$ in the mixture and is reduced $g=G /(n \mathcal{R} T)$ with the universal gas constant $\mathcal{R}$ and the respective phase's overall mole number $n$

$$
g=\sum_{i} x_{i} \mu_{i}(T, p, \boldsymbol{x}) .
$$

The classical framework of irreversible thermodynamics offers to use its concepts locally and to further understand the Gibbs energy as a function of both position and time [42. It has already been demonstrated that local diffusive equilibrium exists across the interphase, which is represented by the equality of the phase's chemical potentials

$$
\mu_{i}^{\mathrm{liq}}=\frac{\partial g^{\mathrm{liq}}}{\partial x_{i}(t)}=\frac{\partial g^{\mathrm{vap}}}{\partial y_{i}(z, t)}=\mu_{i}^{\mathrm{vap}} \quad, \quad i \in[1,2] .
$$

A formulation that is true, due to the extensive Gibbs energy's scaling behavior as a homogeneous function of first-order in the respective mole numbers. The ideal gas acts as the lower physical boundary of every vapor phase given a sufficiently low pressure $p_{0}$. At elevated system pressure $p$, the transition to a real gas mixing behavior leads to

$$
g^{\text {vap }}=\sum_{i=1}^{3} y_{i}\left(\mu_{0, i}^{0}\left(T, p_{0}\right)+\ln \left(\frac{p}{p_{0}}\right)+\ln \left(y_{i}\right)+\ln \left(\varphi_{i}(T, p, \boldsymbol{y})\right)\right),
$$

in which all non-ideal contributions to the chemical potential are summarized into the fugacity coefficient

$$
\varphi_{i}(T, p, \boldsymbol{y})=\psi_{i}(T, p) \vartheta_{i}(T, p, \boldsymbol{y})
$$

that factorizes into a residual $\psi_{i}$ and an excess part $\vartheta_{i}$, fully covering the component's non-ideal behavior and the deviation from ideal mixing at system pressure 
$p$, respectively. This form allows segregating the ideal from the non-ideal mixing contribution to the vapor phase's Gibbs energy

$$
g^{\text {vap }}=\sum_{i=1}^{3} y_{i}\left(\mu_{0, i}(T, p)+\ln \left(y_{i}\right)+\ln \left(\vartheta_{i}(T, p, \boldsymbol{y})\right)\right) .
$$

Liquid phases should be described differently. The components in their pure liquid state at system pressure are chosen to be the reference, while the activity coefficient $\gamma_{i}$ accounts for all non-ideal mixing behavior

$$
g^{\mathrm{liq}}=\sum_{i=1}^{2} x_{i}\left(\mu_{0, i}(T, p)+\ln \left(x_{i}\right)+\ln \left(\gamma_{i}(T, p, \boldsymbol{x})\right)\right) .
$$

The combination of 27, 30 and (31) exposes the correlation between liquid and vapor phase composition, given both are in local equilibrium

$$
y_{i}\left(z_{s}, t\right)=x_{i}(t) \frac{\gamma_{i}(T, p, \boldsymbol{x})}{\vartheta_{i}(T, p, \boldsymbol{y})} .
$$

It is evident from the excess parts' functional dependence on their respective phase composition that a proper substitution of the mole fractions $y_{i}$ to $x_{i}$ requires $\gamma_{i}$ and $\vartheta_{i}$ to be constant, which has already been utilized in 15 . Introducing (32) into 25$)$, together with the diffusion flux's entire thermodynamic contribution

$$
\phi_{i}=\frac{3 \rho_{v} \gamma_{i}}{2 \delta \vartheta_{i}} \mathcal{D}_{i},
$$

leads to the substitution of the vapor phase mole fractions in favor of the liquid phase's.

\subsection{Analytical solution}

A set of non-linear differential equations arises that can be solved by quadrature

$$
d_{t}\left[\begin{array}{l}
x_{1}(t) \rho_{l}(t) z_{l}(t) \\
x_{2}(t) \rho_{l}(t) z_{l}(t)
\end{array}\right]+\left[\begin{array}{cc}
\phi_{1} & 0 \\
0 & \phi_{2}
\end{array}\right] \cdot\left[\begin{array}{l}
x_{1}(t) \\
x_{2}(t)
\end{array}\right] \approx\left[\begin{array}{l}
0 \\
0
\end{array}\right] .
$$

Multicomponent behavior is not inevitably the mere consequence of pure component properties. The simultaneous presence of all species generates excess 
contributions, which can influence the thermal and caloric observables differently. A multicomponent liquid may behave with respect to its thermal properties often times nearly ideal, the density is then a sum of pure component molar densities $\rho_{0, i}$ proportional to the current molar composition $x_{i}(t)$, cf. supplementary material

$$
\rho_{l}(t)^{-1} \approx \sum_{i=1}^{2} x_{i}(t) \rho_{0, i}(T, p)^{-1} .
$$

Vanishing volumetric excess does thereby in no way entail ideal caloric behavior. Applying the derivative and utilizing the assumed ideal behavior (35) reveals a seemingly proper decoupled system

$$
\begin{aligned}
& \rho_{l}^{2} z_{l}\left[\begin{array}{cc}
\rho_{0,2}^{-1} & 0 \\
0 & \rho_{0,1}^{-1}
\end{array}\right] \cdot\left[\begin{array}{c}
\dot{x}_{1} \\
\dot{x}_{2}
\end{array}\right] \\
& +\left[\begin{array}{cc}
\rho_{l} \dot{z}_{l}+\phi_{1} & 0 \\
0 & \rho_{l} \dot{z}_{l}+\phi_{2}
\end{array}\right] \cdot\left[\begin{array}{l}
x_{1} \\
x_{2}
\end{array}\right] \approx\left[\begin{array}{l}
0 \\
0
\end{array}\right],
\end{aligned}
$$

where the liquid's total molar composition enforces a constraint on the mole fractions

$$
x_{1}(t)+x_{2}(t)=1 .
$$

Two thermodynamic parameters can be identified that determine the evaporation rate with the thermodynamic non-ideality quotient $\lambda$ being the more decisive

$$
\epsilon=\frac{\rho_{0,2}}{\rho_{0,1}} \quad, \quad \lambda=\frac{\phi_{2}}{\phi_{1}} .
$$

Multiplying the first line of $[36)$ with $\rho_{0,1}^{-1}$ and the second line with $\rho_{0,2}^{-1}$ allows to fully utilize the constraint (37) and yields upon summation of the resulting equations

$$
\dot{z}_{l}(t)+\rho_{0,2}^{-1} \phi_{2}\left(x_{1}(t)\left(\frac{\epsilon}{\lambda}-1\right)+1\right)=0 .
$$

Similarly, the multiplication of the first line of (36) with $x_{2}(t)$ and the second one with $x_{1}(t)$ yields upon subtraction

$$
\begin{aligned}
& \dot{x}_{1}(t)-\frac{\phi_{2}}{\rho_{0,2} z_{l}(t)}\left(x_{1}^{3}(t)(1-\epsilon)+x_{1}^{2}(t)(\epsilon-2)+x_{1}(t)\right) \\
& \times\left(1-\frac{1}{\lambda}\right)=0 .
\end{aligned}
$$


The film thickness $z_{l}(t)$ has consistently been formulated as a function of time and so has the liquid mole fraction $x_{1}(t)$. The division of $(39)$ by 40 discloses the alternative formulation of film thickness as a function of composition $z_{l}\left(x_{1}(t)\right)$

$$
\frac{d z_{l}(t)}{d x_{1}(t)}=z_{l}(t) \frac{x_{1}(t)\left(1-\frac{\epsilon}{\lambda}\right)-1}{\left(1-\frac{1}{\lambda}\right)\left(x_{1}^{3}(t)(1-\epsilon)+x_{1}^{2}(t)(\epsilon-2)+x_{1}(t)\right)} .
$$

This functional relationship will be resolved successively, where the description of film thickness as a function of composition $z_{l}\left(x_{1}\right)$ is established first and the correlation between mole fraction $x_{1}$ and time $t$ subsequently. It is expedient to factorize the denominator polynomial

$$
\frac{d z_{l}(t)}{d x_{1}(t)}=z_{l}(t) \frac{\frac{1}{1-\epsilon} \frac{\lambda}{\lambda-1}\left(x_{1}(t)\left(1-\frac{\epsilon}{\lambda}\right)-1\right)}{x_{1}(t)\left(x_{1}(t)-1\right)\left(x_{1}(t)-\frac{1}{1-\epsilon}\right)}, \epsilon, \lambda \neq 1,
$$

with the exclusion of pure component behavior, being represented by a mixture of identical particles $(\epsilon, \lambda=1)$. While all denominator roots are evidently real valued, the use of a partial fraction decomposition conveniently partitions the occurring rational function into a primitive sum

$$
\begin{aligned}
\frac{\frac{1}{1-\epsilon} \frac{\lambda}{\lambda-1}\left(x_{1}(t)\left(1-\frac{\epsilon}{\lambda}\right)-1\right)}{x_{1}(t)\left(x_{1}(t)-1\right)\left(x_{1}(t)-\frac{1}{1-\epsilon}\right)} & =\frac{\lambda}{(1-\lambda) x_{1}(t)} \\
& +\frac{1}{(\lambda-1)\left(x_{1}(t)-1\right)} \\
& +\frac{1}{x_{1}(t)-\frac{1}{1-\epsilon}} .
\end{aligned}
$$

A variable separation of 42 in combination with the given decomposition 43 .

renders the emerging equation integrable

$$
\begin{aligned}
\int_{\xi\left(t_{0}\right)}^{\xi(t)} d \xi\left(\frac{1}{\xi(t)}\right)=\int_{x_{1}\left(t_{0}\right)}^{x_{1}(t)} d x_{1} & \left(\frac{\lambda}{(1-\lambda) x_{1}(t)}+\frac{1}{(\lambda-1)\left(x_{1}(t)-1\right)}\right. \\
& \left.+\frac{1}{x_{1}(t)-\frac{1}{1-\epsilon}}\right),
\end{aligned}
$$

with a non-dimensional film thickness $\xi(t)=z_{l}(t) z_{l}\left(t_{0}\right)^{-1}$ that allows to formulate a simpler initial condition

$$
\xi\left(t_{0}\right)=1 \quad, \quad x_{1}\left(t_{0}\right)=x_{1,0} .
$$


An integration reveals the anticipated description of film thickness as a function of composition and unfolds its physical interpretation as being the product of an exponentially weighted composition and liquid density ratio

$$
\xi\left(x_{1}\right)=\left(\frac{x_{1}}{x_{1,0}}\right)^{\frac{\lambda}{1-\lambda}}\left(\frac{x_{2,0}}{x_{2}}\right)^{\frac{1}{1-\lambda}} \frac{\rho_{l}\left(t_{0}\right)}{\rho_{l}(t)} .
$$

To lighten the notation and simplify further calculations, all invariant terms are grouped into one constant $\xi\left(x_{1,0}\right)$ and the liquid density is formulated in terms of molar composition

$$
\xi\left(x_{1}\right)=\xi\left(x_{1,0}\right)\left(\frac{x_{1}^{\lambda}}{1-x_{1}}\right)^{\frac{1}{1-\lambda}}\left(x_{1}-\frac{1}{1-\epsilon}\right) .
$$

The description of liquid composition over time $x_{1}(t)$ cannot easily be constructed from already formulated functions, however, the inverse solution of time as a function of composition $t\left(x_{1}\right)$ is readily attainable

$$
\frac{d \xi\left(x_{1}(t)\right)}{d t}=\frac{d \xi\left(x_{1}(t)\right)}{d x_{1}(t)} \frac{d x_{1}(t)}{d t}
$$

where the derivative on the left hand side is given in $(39)$ and the first derivative on the right hand side can be computed from 47 )

$\frac{d \xi}{d x_{1}}=\xi\left(x_{1,0}\right)\left(x_{1}^{\frac{2 \lambda-1}{1-\lambda}}\left(1-x_{1}\right)^{\frac{\lambda-2}{1-\lambda}}\left(x_{1}+\frac{\lambda}{1-\lambda}\right)\left(x_{1}-\frac{1}{1-\epsilon}\right)+\left(\frac{x_{1}^{\lambda}}{1-x_{1}}\right)^{\frac{1}{1-\lambda}}\right)$.

Equations (39) and (49) contain negligible terms. A reduction in complexity is easily obtainable by exploiting the strongly different volatilities $\lambda \ll 1$ of the two components in the liquid, which physically determines the more volatile component to undergo phase transition preferentially, a characteristic that was built into the particle model used for all simulations

$$
\begin{aligned}
\frac{d \bar{\xi}}{d x_{1}} & \approx \xi\left(x_{1,0}\right)\left(\frac{\left(x_{1}+\lambda\right)\left(x_{1}-\frac{1}{1-\epsilon}\right)}{x_{1}\left(1-x_{1}\right)^{2}}+\frac{1}{1-x_{1}}\right), \\
\frac{d \bar{\xi}}{d t} & \approx \frac{-\phi_{1}}{\rho_{0,2} z_{l, 0}}\left(\epsilon x_{1}+\lambda\right) .
\end{aligned}
$$

The required relationship between composition and time is then determined by an additional separation of variables ansatz in (48) resulting in a quotient 
function

$$
\int_{t_{0}}^{t} d t=\int_{x_{1,0}}^{x_{1}} d x_{1}\left(\frac{d \xi}{d x_{1}} / \frac{d \xi}{d t}\right),
$$

that in combination with the attained approximations $(50 \mathrm{a})$ and $(50 \mathrm{~b})$ generates an analytically solvable integral equation

$$
\begin{aligned}
\int_{t_{0}}^{t} d t=\int_{x_{1,0}}^{x_{1}} d x_{1} & \left(\frac { - \rho _ { 0 , 2 } \xi _ { 0 } z _ { l , 0 } } { \phi _ { 1 } } \left(\frac{\left(x_{1}+\lambda\right)\left(x_{1}-\frac{1}{1-\epsilon}\right)}{x_{1}\left(1-x_{1}\right)^{2}\left(\epsilon x_{1}+\lambda\right)}\right.\right. \\
& \left.\left.+\frac{1}{\left(1-x_{1}\right)\left(\epsilon x_{1}+\lambda\right)}\right)\right)
\end{aligned}
$$

where the emerging rational function similarly partitions into a primitive sum, given all denominator roots being real valued

$$
\begin{aligned}
\frac{\left(x_{1}+\lambda\right)\left(x_{1}-\frac{1}{1-\epsilon}\right)}{x_{1}\left(1-x_{1}\right)^{2}\left(\epsilon x_{1}+\lambda\right)} & =\frac{a_{1}(\epsilon, \lambda)}{x_{1}}+\frac{a_{2}(\epsilon, \lambda)}{1-x_{1}} \\
& +\frac{a_{3}(\epsilon, \lambda)}{\left(1-x_{1}\right)^{2}}+\frac{\epsilon a_{4}(\epsilon, \lambda)}{\epsilon x_{1}+\lambda}, \\
\frac{1}{\left(1-x_{1}\right)\left(\epsilon x_{1}+\lambda\right)} & =\frac{a_{5}(\epsilon, \lambda)}{1-x_{1}}+\frac{\epsilon a_{6}(\epsilon, \lambda)}{\epsilon x_{1}+\lambda} .
\end{aligned}
$$

All occurring integration constants show the already proclaimed dependence on the thermodynamic parameters $\epsilon, \lambda$ and do thereby significantly contribute to the film's evaporation rate

$$
\begin{aligned}
& a_{1}(\epsilon, \lambda)=\frac{1}{\epsilon-1}, a_{2}(\epsilon, \lambda)=\frac{\lambda \epsilon^{2}+\epsilon+\lambda+\lambda^{2}}{(\epsilon-1)(\epsilon+\lambda)^{2}}, \\
& a_{3}(\epsilon, \lambda)=\frac{\lambda \epsilon+\epsilon}{(\epsilon-1)(\epsilon+\lambda)}, a_{4}(\epsilon, \lambda)=\frac{\lambda \epsilon-\epsilon-\lambda}{(\epsilon+\lambda)^{2}} \\
& a_{5}(\epsilon, \lambda)=a_{6}(\epsilon, \lambda)=\frac{1}{\epsilon+\lambda} .
\end{aligned}
$$

As a result, the functional relationship between composition and time is decomposed into four linear terms that are straightforwardly integrable to yield

$$
\begin{aligned}
t\left(x_{1}\right) & =\frac{\rho_{0,2} \xi_{0} z_{l, 0}}{\phi_{1}}\left(a_{1} \ln \left(\frac{x_{1,0}}{x_{1}}\right)+a_{7} \ln \left(\frac{x_{2}}{x_{2,0}}\right)\right. \\
& \left.+a_{3} \frac{x_{2}-x_{2,0}}{x_{2} x_{2,0}}+a_{8} \ln \left(\frac{\epsilon x_{1,0}+\lambda}{\epsilon x_{1}+\lambda}\right)\right)
\end{aligned}
$$


with $a_{7}=a_{2}+a_{5}$ and $a_{8}=a_{4}+a_{6}$.

The solution of a liquid film's Stefan problem, i.e. the d-squared law's (1) planar surface analogue for both cases, the two-component and pure liquid phase is readily obtained. The former is given by the combination of 47 and 55 relating film thickness $\xi\left(x_{1}\right)$ to time $t\left(x_{1}\right)$ via the liquid's mole fraction $x_{1}$. The latter is a special case for $\epsilon, \lambda=1$, that is not entailed in (47) and (55), yet is alternatively obtained by integration of $(39)$ with the addition of 32$)$ to substitute the vapor phase's non-ideality $\vartheta_{i}$ with the evaporate's mole fraction $y_{e, s}$ near the surface

$$
z_{l}(t)=z_{l, 0}-\frac{3 D \rho_{v}}{2 \delta \rho_{l}} y_{e, s} t
$$

Comparing pure component liquid films to droplets offers a salient difference in the order to which both cases evaporate with respect to their characteristic length $z_{l}(t)$ and $d(t)$. In this first-order approximation (56) the liquid film evaporates linearly in time, where the spherical droplet's analogue for the same level of approximation, as given by Maxwell's equation (2), regresses quadratically

$$
d^{2}(t)=d_{0}^{2}-\frac{8 D \rho_{v}}{\rho_{l}} y_{e, s} t
$$

A high evaporation rate, i.e. short liquid phase lifetime, is facilitated by a high diffusivity or low liquid density, respectively. For planar surfaces, the boundary layer thickness $\delta$ remains in the description as a finite size effect, which is in full accordance with classical boundary layer theory. Small values of $\delta$ correspond to steep chemical potential gradients that inevitably lead to faster evaporation 135 rates.

\section{Results}

Under vapor-liquid equilibrium (VLE), the mass fluxes between both bulk phases balance and consequently no net mass transfer occurs. During evaporation, the liquid film is constantly forfeiting mass in the attempt to dispose of the developed chemical potential gradient and restore a VLE. The establishing evaporation dynamics affects each bulk phase's molar composition differently. 
Due to the volatile component's characteristic to evaporate preferentially, the less-volatile component successively enriches within the liquid, as depicted in figure 3 for the interphase's vicinity at $z=z_{l}(t)$. The process causes the film not only to regress but also increases its overall density, since the less-volatile component has a higher saturated liquid density, cf. table 1 and figure 4 . The bulk vapor phase's molar composition in vicinity to the interphase does marginally change during evaporation. While the volatile component depletes quite rapidly, the inert gas accumulates at the surface. The less-volatile's mole fraction, in contrast, remains almost stagnant, cf. figure 5 evaluated at $z=z_{s}(t)$.

The combination of (47) and (55) allows to compute a two-component film's dimensionless thickness $\xi(t)$ as function of time, if the phases' non-idealities $\vartheta_{i}, \gamma_{i}$ and the components' Fick diffusion coefficients $D_{i j}$ are known. Similarly, (56) determines a monocomponent film's regression rate, given the evaporate's diffusion coefficient $D$ and molar composition adjacent to the interphase $y_{e, s}$ are known. All required thermodynamic data were sampled by molecular simulations, cf. Appendix A and are listed in table 1, Appendix B and the enclosed supplementary material. Three differently composed liquid films were investigated. Ranging from pure volatile over an initially equimolarly composed two-component mixture to pure less-volatile. The monocomponent films' evaporation rates expectedly envelop those of all possible binary mixtures, cf. figure 6. The equimolarly prepared film regresses initially quite rapidly and then, due to a limitation embedded in (55) that predicts the volatile component's full evaporation to be reached only asymptotically $\lim _{x_{1} \rightarrow 0} t\left(x_{1}\right)=\infty$, transitions into an apparently stagnant and finite thickness for small values of $x_{1}$, i.e. $x_{1} \leq 0.01 \mathrm{~mol} \mathrm{~mol}^{-1}$. In contrast, the large scale MD simulations indicate a transition into a constant rate that closely resembles, yet not equals, the pure less-volatile component's evaporation rate $K(T, p, \boldsymbol{x}) \approx K_{2}(T, p)$, since residual volatile particles still remain in the vapor phase for an extended period of time. 


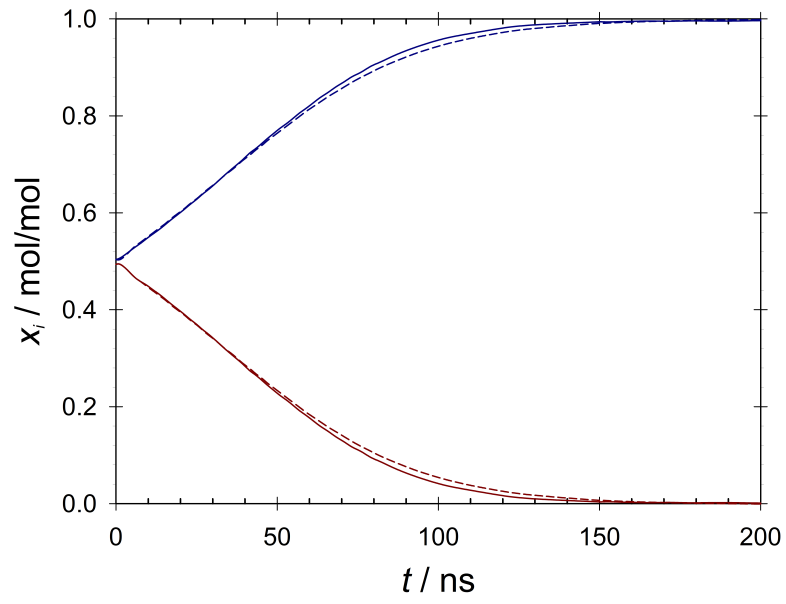

Figure 3: Time evolution of the molar fractions of the volatile (red) and less-volatile (blue) components in the liquid phase. The dashed lines represent the analytical solution and the solid lines are large scale MD simulation data, evaluated on the liquid side of the interphase at $z_{l}(t)$. The liquid is successively enriched with the less-volatile component.

\section{Conclusion}

The Stefan problem for a multicomponent planar film was solved. An analytical model was presented, describing evaporation dynamics of a liquid film into a dense non-ideal vapor phase. The outlined formalism requires specified bulk phase non-idealities $\gamma_{i}, \vartheta_{i}, \psi_{i}$, as well as Fick diffusion coefficients $D_{i j}$ at the interphase's vicinity, i.e. at $z=z_{s}(t)$. Consequently, its solutions (47), (55) and (56) allow to determine the time evolution of both phases' molar compositions $\boldsymbol{x}(t), \boldsymbol{y}(t)$ as well as the film's thickness $\xi(t)$ quantitatively. The components' deliberately specified characteristics, i.e. strongly different volatilities $\lambda \ll 1$, weak diffusion flux coupling $\mathbf{T} \approx \mathbf{I}$ and the inert gas behavior, facilitate expedient simplifications in the derivation of the model and its solutions.

The limiting quantity of a two-component film's evaporation rate $\dot{\xi}(t)$ is identified as the volatile component's diffusion flux $\mathcal{L}_{z, 1}$, wherein all driving influences, i.e. the activity coefficient $\gamma_{1}$, the fugacity coefficient's excess contribution $\vartheta_{1}$, the vapor phase thickness $\delta$ and the effective diffusivity $\mathcal{D}_{1}$, appear 


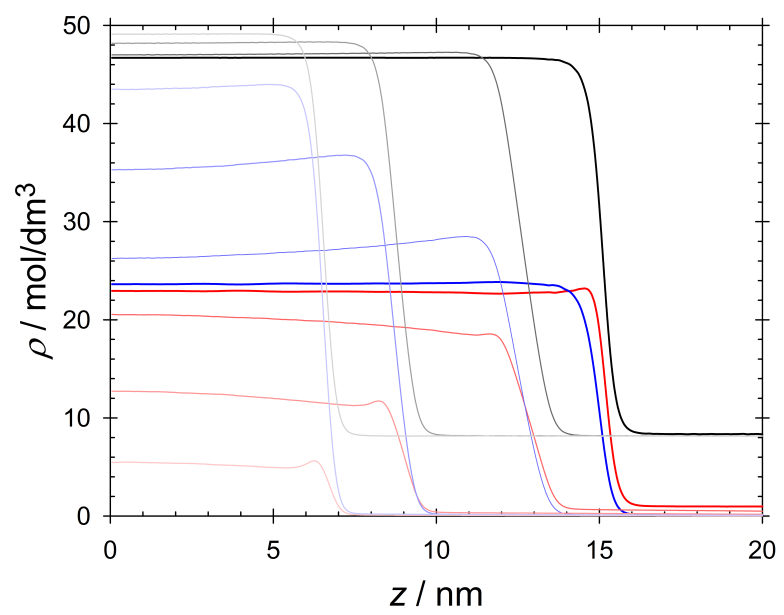

Figure 4: Time evolution of the partial density profiles of both components in the liquid mixture as sampled by the present large scale MD simulations. Two opposing density gradients emerge, resulting in two counter-oriented currents, where the volatile component (red,1) propagates towards the interphase and preferentially evaporates, while the less-volatile component (blue,2) evaporates at a lower rate and partly regresses towards the liquid film's center. A local volatile component's enrichment within the interphase is observable. The respective overall density is depicted in black. The liquid film's density increase over time is a mixture effect due to the enrichment of component 2 that has a higher saturated liquid density $\left(\rho_{l, 1}<\rho_{l, 2}\right)$.

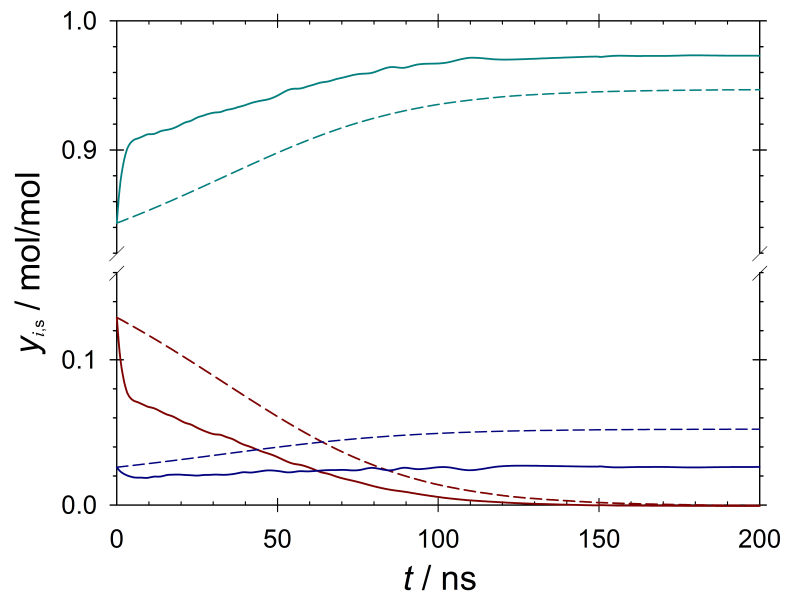

Figure 5: Time evolution of the vapor phase mole fraction on the vapor side of the interphase at $z_{s}(t)$, where the dominating inert gas' behavior (cyan) is prominent. 


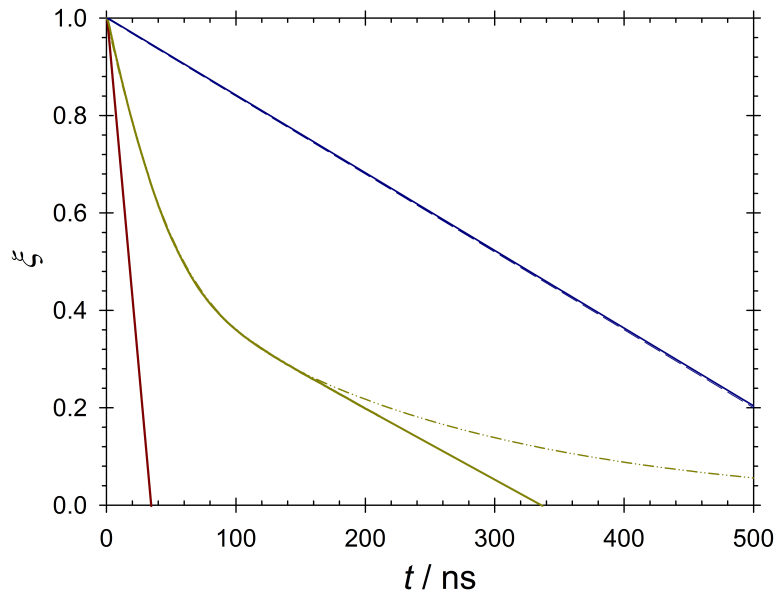

Figure 6: Time evolution of dimensionless film thickness $\xi(t)=z_{l}(t) / z_{l}\left(t_{0}\right)$, where the dotted lines represent the analytical solution while the solid lines the large scale MD simulations. The evaporation rate is given by the slope of these curves, where the equimolar liquid case (yellow) is enveloped by the pure volatile's (red) and pure less-volatile's (blue) rate $K_{1}(T, p)>$ $K(T, p, \boldsymbol{x})>K_{2}(T, p)$. The strong difference between the evaporation rates of the two pure fluids $K_{1}, K_{2}$ is intentional and a consequence of the chosen intermolecular force field model. 
185

linearly and consequently affect the film's behavior equally.

The analytical model was intentionally contrasted to large scale MD simulations serving three purposes. First, the phases' molar compositions were assessed in an unconstrained manner. Second, the existence of local thermodynamic equilibrium was verified unambiguously in every domain and third it was demonstrated that the hydrodynamic formalism can justifiably be applied to such a nanoscale scenario. The MD simulations fully confirm that neglecting the film's internal dynamics, by assuming spatial homogeneity $x_{i} \neq x_{i}(z)$, as in (6) and (7), does not compromise the accurate prediction of its composition $\boldsymbol{x}(t)$ and thickness $\xi(t)$ over time. Consequently, the hydrodynamic field description is applicable to system sizes of the order of $50 \mathrm{~nm}$, given the thermophysical properties are known a priori. Atomistic simulations prove to be an adequate methodology determining all required properties without constraints. The symbiosis of both approaches offers a promising route for further studies on evaporation.

\section{Supplementary material}

All calculated and sampled data are disclosed in this manuscript's supplementary material.

\section{Acknowledgements}

The present work contributes to the Collaborative Research Center (SFB) 75 of Deutsche Forschungsgemeinschaft (DFG) and was funded under the grant VR 6/9-2. All computations were performed either on the HPC cluster OCuLUS at the Paderborn Center for Parallel Computing $\left(\mathrm{PC}^{2}\right)$ or on the Cray XC40 system Hazel Hen at the High Performance Computing Center Stuttgart (HLRS) with resources allocated according to grant MMHBF2. This work was carried out 


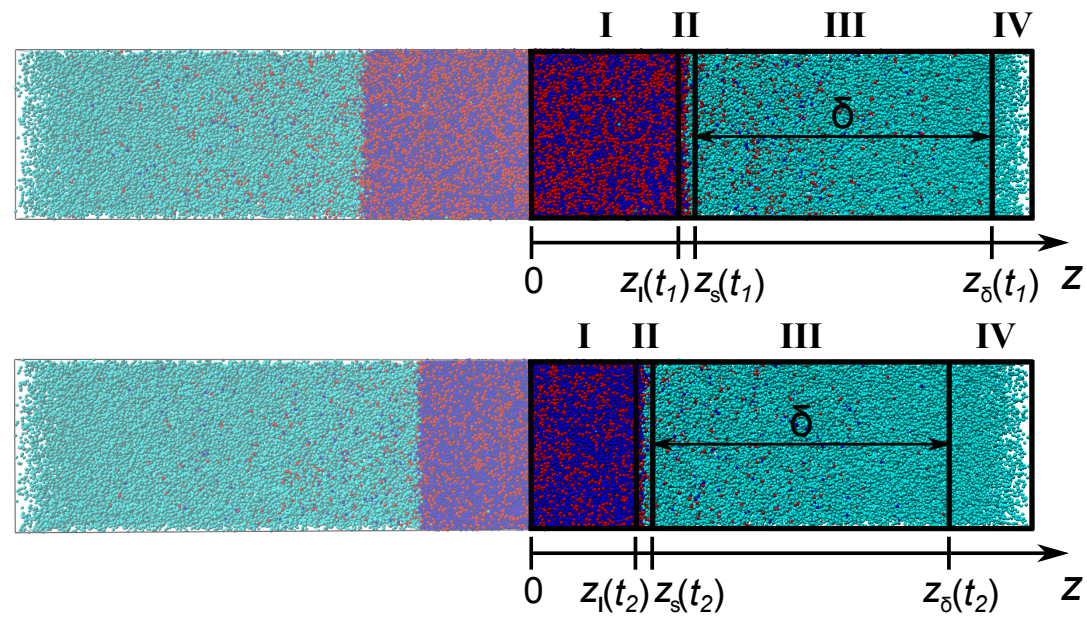

Figure A.7: Snapshot taken from the present large scale MD simulations depicting two successive instants of time $t_{2}>t_{1}$ during evaporation. While the liquid film (I) regresses, the control volume (IV) was simultaneously expanded to keep the vapor phase's (III) thickness constant $\delta \neq \delta(t)$.

\section{Appendix A. Molecular dynamics simulations}

The model fluid's thermodynamic behavior is determined by deliberately specified Lennard-Jones parameters as outlined in table A.2. The component's distinct volatilities were realized here by different energy parameters $\epsilon_{i}$. The in215 ert gas characteristic to be barely miscible in the liquid was modeled primarily by the Berthelot parameter $\zeta_{i 3} \neq 1$.

All particles in the investigated scenario interact via the full Lennard-Jones potential with $3.5 \sigma$ cut-off radius and analytic long range corrections [43]. The chosen Lennard-Jones parameters approximately describe a liquid film composed of either nitrogen + oxygen or argon + krypton. The large scale MD simulations were carried out in a cuboid volume with dimensions $L_{x}=L_{y}=15$ $\mathrm{nm}$ and $L_{z}=90 \mathrm{~nm}$, an integrator time step of $\Delta t=5 \mathrm{fs}$ and periodic boundary conditions in $x$ and $y$ directions.

The present large scale MD simulations are, technically speaking, nonequilibrium molecular dynamics (NEMD) simulations, which does not inevitably lead to non-Maxwellian distributed particle velocities, but allows possible local 


\begin{tabular}{lcccccc}
\hline component & $i$ & $\sigma_{i} / \mathrm{nm}$ & $\epsilon_{i} / \mathrm{k}_{B} / \mathrm{K}$ & $m_{i} / \mathrm{u}$ & $\zeta_{1 i} /-$ & $\zeta_{2 i} /-$ \\
\hline volatile & 1 & 0.3 & 75 & 2 & - & 1 \\
less-volatile & 2 & 0.3 & 100 & 20 & 1 & - \\
inert gas & 3 & 0.3 & 10 & 30 & 0.3 & 0.3 \\
\hline
\end{tabular}

Table A.2: The thermodynamic behavior of the fluid sampled by simulation was determined by this set of Lennard-Jones parameters that were chosen to produce a strongly non-ideal vapor, yet retain a fairly ideal liquid phase. The length parameter $\sigma$ determines a particle's effective diameter while the energy parameter $\epsilon$ is a measure for a particle's dispersive interaction strength. Additionally, a modification of the Berthelot combining rule via $\zeta_{k i}$ allows to alter the interaction energy between unlike particle species. The inert gas was in a highly supercritical state and consists of mainly repulsive interacting heavy particles. While all simulations sampling thermodynamic observables were carried out with the open source molecular simulation tool $m s 2$ [4], the large scale MD calculations were performed with the massively parallel open source code $l s 1$ mardyn 45 .

equilibria to arise naturally, as demonstrated in section 2.1. In order to investigate evaporation dynamics that are driven exclusively by a spatial chemical potential gradient the system was selectively thermostated. Consequently, the less-volatile particles in the liquid $z \leq z_{l}(t)$ and the inert gas particles in the vapor $z \in\left[z_{s}(t), z_{\delta}(t)\right]$ were thermostated in $x$ and $y$ directions only, avoiding artificial interference with the investigated mass transport in the relevant $z$ direction.

The necessary concentration gradient to initiate and maintain evaporation dynamics was realized by substituting all particles of components 1 and 2 by inert gas particles (component 3) within the control volume, i.e. maintaining a molar composition of $y_{3}=1$ at $z>z_{\delta}(t)$. During evaporation, the control volume was expanded to the same extent as the liquid film regressed, keeping the vapor phase thickness $\delta \neq \delta(t)$ constant, cf. figure A.7.

The symmetry of the fluid system was exploited to generate considerably smoother sampling profiles by calculating the arithmetic mean between both sides' density and molar composition profiles. 


\section{Appendix B. Diffusion properties}

A thermodynamically non-ideal system can display a conspicuous disparity between multicomponent and collective pure component behavior, as measured by the Gibbs energy's excess contribution

$$
g^{e}(T, p, \boldsymbol{y})=\sum_{i=1}^{3} y_{i} \ln \left(\vartheta_{i}\right) .
$$

The fugacity coefficient's excess contribution $\vartheta_{i}$ can alternatively be calculated from the species' chemical potentials $\mu_{i}$ Appendix Cl and in contrast to the conventional approach, utilizing equations of state, were computed here via dedicated MC simulations

$$
\ln \left(\vartheta_{i}\right)=\mu_{i}-\ln \left(y_{i}\right)-\mu_{0, i}
$$

Non-ideal behavior, however, is not merely described by the excess Gibbs en-

245 ergy's numerical value but also by its derivatives [46], where the thermodynamic factor is determined by the curvature

$$
\begin{aligned}
\Gamma_{i j}=\delta_{i j}+y_{i} & \left(\frac{\partial g^{e}}{\partial y_{j} \partial y_{i}}-\frac{\partial g^{e}}{\partial y_{i} \partial y_{3}}\right. \\
& \left.-\sum_{k=1}^{3} y_{k}\left(\frac{\partial g^{e}}{\partial y_{k} \partial y_{j}}-\frac{\partial g^{e}}{\partial y_{k} \partial y_{3}}\right)\right) .
\end{aligned}
$$

A discrete data set of $g^{e}$ values for a varying molar composition $\boldsymbol{y}=\left(y_{1}, y_{2}, y_{3}\right)$ was produced by comprehensive MC simulations (see supplementary material) and the computation of the necessary derivatives in B.3 was made possible by selecting an appropriate $g^{e}$ model. An appropriate ansatz is a Margules type polynomial that is the result of an empirically motivated Wohl's expansion 47. truncated to desired order. As data generated by simulations were made sufficiently precise and plentifully available, an advanced fourth-order ansatz 
[48] became feasible

$$
\begin{aligned}
g^{e} & =y_{1} y_{2} y_{3}\left(A_{12}+A_{21}+A_{32}-C_{1} y_{1}-C_{2} y_{2}-C_{3} y_{3}\right) \\
& +y_{1} y_{2}\left(A_{21} y_{1}+A_{12} y_{2}-E_{12} y_{1} y_{2}\right) \\
& +y_{1} y_{3}\left(A_{31} y_{1}+A_{13} y_{3}-E_{13} y_{1} y_{3}\right) \\
& +y_{2} y_{3}\left(A_{32} y_{2}+A_{23} y_{3}-E_{23} y_{2} y_{3}\right)
\end{aligned}
$$

where the first term models the genuine ternary contribution with parameters $C_{i}$ and the following three terms describe the respective binary subsystems' influence with parameters $A_{i j}, E_{i j}$. All parameters $\left(A_{i j}, E_{i j}, C_{i}\right)$ were determined with a standard nonlinear least squares fit to the respective excess Gibbs energy data obtained from MC simulations.

The Fick diffusion matrix $\mathbf{D}$ can be decomposed into a kinetic $\mathbf{B}^{-1}$ and a thermodynamic $\boldsymbol{\Gamma}$ contribution, which are functions of the vapor phase's molar composition. It has been argued above that both matrices were considered constant and will only be evaluated at equilibrium condition $\boldsymbol{y}^{*}=(0.13,0.03,0.84)$ $\mathrm{mol} \mathrm{mol}-1$

$$
\boldsymbol{\Gamma}\left(\boldsymbol{y}^{*}\right)=\left[\begin{array}{cc}
0.82 & -0.18 \\
-0.03 & 1.0
\end{array}\right] \text {. }
$$

${ }_{255}$ Determining the kinetic contribution $\mathbf{B}^{-1}$, however, is a two-step procedure. The Maxwell-Stefan diffusivities $\bigoplus_{i j}$ have to be computed first, which for this ternary mixture are readily accessible via MD simulations

$$
\begin{aligned}
& \bigoplus_{13}=171.0 \cdot 10^{-9} \mathrm{~m}^{2} \mathrm{~s}^{-1} \\
& \bigoplus_{23}=58.0 \cdot 10^{-9} \mathrm{~m}^{2} \mathrm{~s}^{-1} \\
& \bigoplus_{12}=34.5 \cdot 10^{-9} \mathrm{~m}^{2} \mathrm{~s}^{-1}
\end{aligned}
$$

and subsequently the matrix $\mathbf{B}$ with its diagonal $B_{i i}$ and off-diagonal elements $B_{i j}$ has to be calculated [39]

$$
\begin{aligned}
B_{i i} & =\frac{y_{i}}{\mathrm{Ð}_{i 3}}+\sum_{k=1 \neq i}^{3} \frac{y_{i}}{\mathrm{Ð}_{i k}}, \\
B_{i j} & =-y_{i}\left(\frac{1}{\mathrm{Ð}_{i j}}-\frac{1}{\mathrm{Ð}_{i 3}}\right), i, j \in[1,2] .
\end{aligned}
$$


The Maxwell-Stefan diffusivities $\bigoplus_{i j}$ quantify the relative motion between the $i$ th and $j$-th component in the ternary mixture and are incorporated reciprocally in the description. Consequently, it is this matrix's inverse that is part of the thermodynamic contribution to relate the driving thermodynamic force, i.e. the concentration gradient to its corresponding diffusion flux, and is given as

$$
\mathbf{B}^{-1}\left(\boldsymbol{y}^{*}\right)=\left[\begin{array}{cc}
155.78 & 2.62 \\
25.02 & 53.69
\end{array}\right] \cdot 10^{-9} \mathrm{~m}^{2} \mathrm{~s}^{-1} .
$$

The Fick diffusion matrix $\mathbf{D}=\mathbf{B}^{-1} \cdot \boldsymbol{\Gamma}$ couples the simultaneous influence of both gradients to each molar diffusion flux $\mathcal{L}_{z, i}$, as specified in 13). A spectral decomposition facilitates the decoupling by mapping both gradients' influence onto an effective diffusion coefficient $\mathcal{D}_{i}$ that is given as the system's $i$-th eigenvalue

$$
\mathcal{D}\left(\boldsymbol{y}^{*}\right)=\left[\begin{array}{cc}
120.97 & 0 \\
0 & 55.88
\end{array}\right] \cdot 10^{-9} \mathrm{~m}^{2} \mathrm{~s}^{-1},
$$

where the deviation of the eigenvector matrix $\mathbf{T}$ from the identity matrix $\mathbf{I}$ is a measure of coupling strength and the arguably present mild coupling is neglected by setting

$$
\mathbf{T}=\left[\begin{array}{cc}
1 & 0.35 \\
0.26 & 1
\end{array}\right] \approx \mathbf{I}
$$

The simplification introduced in 50a and $50 \mathrm{~b}$ was based on the components' strongly different volatilities $\lambda \ll 1$ and the exclusion of a pseudo mixture $\epsilon \neq 1$

$$
\lambda=\frac{\phi_{2}}{\phi_{1}}=0.093, \epsilon=\frac{\rho_{0,2}}{\rho_{0,1}}=1.18 .
$$

\section{Appendix C. Thermodynamic non-ideality}

The vapor phase's Gibbs energy could be decomposed into the sum of a variety of physically interpretable contributions

$$
g^{\mathrm{vap}}(T, p, \boldsymbol{y})=\sum_{i=1}^{3} g_{0, i}^{0}(T, p)+g_{0, i}^{\mathrm{res}}(T, p)+y_{i} \ln \left(y_{i}\right)+g_{i}^{e}(T, p, \boldsymbol{y}),
$$


with the first term being the ideal gas contribution of the pure component, given at system temperature and pressure, and the second its residual contribution. The third term describes the ideal mixing term and the fourth its non-ideal mixing correction. The Gibbs energy can also be decomposed into the weighted sum of its components' chemical potentials, as stated in 26 .

$$
g^{\operatorname{vap}}(T, p, \boldsymbol{y})=\sum_{i=1}^{3} y_{i} \mu_{i}(T, p, \boldsymbol{y})
$$

The ideal gas contribution is readily understood as the ideal gas chemical potential $g_{0, i}^{0}=y_{i} \mu_{0, i}^{0}$. The residual and excess contribution's mathematical form has, historically arisen, been defined to be a weighted logarithmic function akin to the ideal gas mixing entropy

$$
\begin{aligned}
g^{\mathrm{vap}}(T, p, \boldsymbol{y})=\sum_{i=1}^{3} \quad y_{i}\left(\mu_{0, i}^{0}(\right. & T, p)+\ln \left(\psi_{i}(T, p)\right)+\ln \left(y_{i}\right) \\
+ & \left.\ln \left(\vartheta_{i}(T, p, \boldsymbol{y})\right)\right)
\end{aligned}
$$

Generally, all pure component contributions were grouped together to ease notation

$$
\mu_{0, i}(T, p)=\mu_{0, i}^{0}(T, p)+\ln \left(\psi_{i}(T, p)\right),
$$

which consequently leads to the vapor phase's Gibbs energy

$$
g^{\operatorname{vap}}(T, p, \boldsymbol{y})=\sum_{i=1}^{3} y_{i}\left(\mu_{0, i}(T, p)+\ln \left(y_{i}\right)+\ln \left(\vartheta_{i}(T, p, \boldsymbol{y})\right)\right) .
$$

Bringing (C.2) and (C.5) together leads to description $(\mathrm{B} .2)$ for the case of known chemical potentials $\mu_{i}$, which were sampled by here by dedicated MC simulations

$$
\ln \left(\vartheta_{i}\right)=\mu_{i}-\ln \left(y_{i}\right)-\mu_{0, i}
$$

Alternatively, if the mixture's Gibbs excess energy is known, e.g. from a $g^{e}$ model, the component's excess contribution to the fugacity coefficient can be computed [46]

$$
\ln \left(\vartheta_{i}\right)=g^{e}+\frac{\partial g^{e}}{\partial y_{i}}-\sum_{k=1}^{3} y_{k} \frac{\partial g^{e}}{\partial y_{k}}
$$




\section{References}

[1] C. Law, Recent advances in droplet vaporization and combustion, Energy Combust. Sci. 8 (1982) 171-201.

[2] H. Morse, On evaporation from the surface of a solid sphere. Preliminary note, Proc. Amer. Acad. Sci. 45 (1910) 363-367.

[3] N. Fuchs, Über die Verdampfungsgeschwindigkeit kleiner Tröpfchen in einer Gasatmosphäre, Phys. Z. Sowjetunion 6 (1934) 224-243.

[4] J. C. Maxwell, in: W. D. Niven (Ed.), The Scientific Papers of James Clark Maxwell, Vol. 2, Cambridge Libarary Collection, 2011, pp. 625-646.

[5] N. Fuchs, Evaporation and Droplet Growth in Gaseous Media, Pergamon Press, 1959.

[6] D. Spalding, The combustion of liquid fuels, Fourth Symp. of Comb. 4 (1953) 847-864.

[7] S. Kumagai, T. Sakai, S. Okajima, Combustion of free fuel droplets in a freely falling chamber, Proc. Combust. Inst. 13 (1971) 779-785.

[8] S. Okajima, S. Kumagai, Further investigations of combustion of free droplets in a freely falling chamber including moving droplets, Proc. Combust. Inst. 15 (1975) 401-407.

[9] C. T. Avedisian, J. C. Yang, C. H. Wang, On low-gravity droplet combustion, Proc. R. Soc. Lond. 420 (1988) 183-200.

[10] I. Gökalp, C. Chauveau, J. Richard, M. Kramer, W. Leuke, Observations on the low temperature vaporization and envelope or wake flame burning of n-heptane droplets at reduced gravity during parabolic flights, Proc. Combust. Inst. 22 (1989) 2027-2035.

[11] C. Chauveau, I. Gökalp, Droplet combustion under microgravity conditions, in: B. Kaldreich (Ed.), VIIIth European Symposium on Materials 
and Fluid Sciences in Microgravity, Vol. 2, European Space Agency, Paris, 1992, pp. 467-472.

[12] C. Chauveau, X. Chesnau, I. Gökalp, Burning characteristics of n-heptane droplets under different regimes, in: 31st Aerospace Science Meeting, 1993, pp. $824-832$.

[13] H. Nomura, Y. Ujiie, H. J. Rath, J. Sato, M. Kono, Experimental study on high-pressure droplet evaporation using microgravity conditions, Proc. Combust. Inst. 26 (1996) 1267-1273.

[14] V. Nayagam, J. B. Haggard, R. O. Colantonio, A. J. Marchese, F. L. Dryer, B. L. Zhang, F. A. Williams, Microgravity n-heptane droplet combustion in oxygen-helium mixtures at atmospheric pressure, AIAA J. 36 (1998) $1369-1378$.

[15] C. Morin, C. Chaveau, I. Gökalp, Droplet vaporization characteristics of vegetable oil derived biofuels at high temperatures, Exp. Therm Fluid. Sci. 21 (2000) 41-50.

[16] H. Ghassemi, S. W. Baek, Q. S. Khan, Experimental study on evaporation of kerosine droplets at elevated pressures and temperature, Combust. Sci. Technol. 178 (2006) 1669-1684.

[17] V. Glushkov, O. Todes, V. Fedoseev, Evaporation kinetics of drops of binary mixtures of organic liquids, Kolloidn Zh. 32 (1967) 678-683.

[18] E. Ravindran, J. Davis, Multicomponent evaporation of single aerosol droplets, J. Coll. Int. Sci. 85 (1982) 278-288.

[19] G. Brenn, L. Deviprasath, F. Durst, C. Fink, Evaporation of acoustically levitated multi-component liquid droplets, Int. J. Heat Mass Transf. 50 (2007) 5073-5086.

[20] S. Sumardiono, J. Fischer, Molecular dynamics simulations of mixture droplet evaporation, in: J. Pagliarini, S. Rainieri (Eds.), Eurotherm Sem- 
inar 77, Heat and mass transfer in food processing, Edizione ETS Parma, Parma, 2005, p. 323.

[26] R. Schunk, A. Nagy, Ionospheres: Physics, Plasma Physics, and Chemistry, Cambridge University Press, 2009.

[27] B. Hafskjold, S. Kjelstrup, Criteria for local equilibrium in a system with transport of heat and mass, J. Stat. Phys. 78 (1995) 463-494.

[28] I. Inazoli, S. Kjelstrup, D. Bedeaux, J. Simon, Thermodynamic properties of a liquid-vapor interface in a two-component system, Chem. Eng. Sci. 65 (2010) 4105-4116.

[29] J. Xu, S. Kjielstrup, D. Bedeaux, A. Røsjorde, L. Rekvig, Verification of Onsager's reciprocal relations for evaporation and condensation using nonequilibrium molecular dynamics, J. Colloid Interface Sci. 299 (2006) 452463.

[30] S. Kjelstrup, D. Bedeaux, I. Inazoli, J.-M. Simon, Criteria for validity of thermodynamic equations from non-equilibrium molecular dynamics simulations, Energy 33 (2008) 1185-1196. 
345 [31] T. Ishiyama, T. Yano, S. Fujikawa, Molecular dynamics study of kinetic boundary condition at an interface between argon vapor and its condensed phase, Phys. Fluids 16 (2004) 2899-2906.

[32] A. Frezzotti, L. Gibelli, S. Lorenzani, Mean field kinetic theory description of evaporation of a fluid into vacuum, Phys. Fluids 17 (2005) 012102.

350 [33] A. Frezzotti, Boundary conditions at the vapor-liquid interface, Phys. Fluids 23 (2011) 030609.

[34] L. D. Landau, E. Lifshitz, Course of Theoretical Physics. Fluid Mechanics, Pergamon Press, 1987.

[35] S. de Groot, P. Mazur, Non-Equilibrium Thermodynamics, Dover Press, 1984.

[36] B. van Milligen, P. Bons, B. Carreras, R. Sánchez, On the applicability of Fick's law to diffusion in inhomogeneous systems, Eur. J. Phys. 26 (2005) 913-925.

[37] A. Fick, Über Diffusion, Ann. Phys. 170 (1855) 59-86.

[38] C. Truesdell, Mechanical basis of diffusion, J. Chem. Phys. 37 (1962) 23362344.

[39] R. Taylor, R. Krishna, Multicomponent Mass Transfer, Wiley, 1993.

[40] K. Pohlhausen, Zur näherungsweisen Integration der Differentialgleichung der laminaren Grenzschicht, ZAMM 1 (1921) 252-290.

365 [41] D. Spalding, Mass transfer in laminar flow, Proc. R. Soc. A 221 (1954) 78-99.

[42] G. Lebon, D. Jou, J. Casas-Vázquez, Understanding Non-Equilibrium Thermodynamics, Springer, 2008.

[43] J. Janeček, Long range corrections in inhomogeneous simulations, J. Phys. Chem. B 110 (2006) 6264-6269. 
[44] C. W. Glass, S. Reiser, G. Rutkai, S. Deublein, A. Köster, G. GuevaraCarrion, A. Wafai, M. Horsch, M. Bernreuther, T. Windmann, H. Hasse, J. Vrabec, ms2: A molecular simulation tool for thermodynamic properties, new version release, Comp. Phys. Commun. 185 (2014) 3302-3306.

[45] C. Niethammer, S. Becker, M. Bernreuther, M. Buchholz, W. Eckhardt, A. Heinecke, S. Werth, H. Bungartz, C. Glass, H. Hasse, J. Vrabec, M. Horsch, ls1 mardyn: The massively parallel molecular dynamics code for large systems, J. Chem. Theory Comput. 10 (2014) 4455-4464.

[46] R. Taylor, H. Koojiman, Composition derivatives of activity coefficient models (for the estimation of thermodynamic factors in diffusion), Chem. Eng. Commun. 102 (1991) 87-106.

[47] J. Prausnitz, R. Lichtenthaler, E. de Azvedo, Molecular Thermodynamics of Fluid-Phase Equilibria, 3rd Edition, Prentice Hall, 1999.

[48] E. Hála, J. Pick, V. Fried, O. Vilím, Vapor-Liquid Equilibrium, Pergamon Press, 1967. 


\title{
Diffusion limited evaporation of a multicomponent liquid film
}

\author{
René Spencer Chatwell, Matthias Heinen, and Jadran Vrabec ${ }^{1, a)}$
}

This supplementary material discloses all calculated thermodynamic data for the present analytical solution together with the $g^{e}$ model relying on data sampled by $\mathrm{MD}$ or MC simulations. Values from the analytical solution are indicated by the subscript ana, values from the $g^{e}$ model are denoted by mod and simulated data are specified by sim. In the following tables, all quantities are understood as functions of either liquid $\left[x_{i}\right]=\mathrm{mol}$ $\mathrm{mol}^{-1}$ or vapor phase $\left[y_{i}\right]=\mathrm{mol} \mathrm{mol}{ }^{-1}$ mole fractions, if not otherwise specified. The Gibbs excess energy $g^{e}$ and all chemical potentials $\mu_{i}$ are given in non-dimensional form, i.e. $g^{e}=G^{e} / n \mathcal{R} T$ and $\mu_{i}=\bar{\mu}_{i} / \mathcal{R} T$

All time-independent thermodynamic properties were determined by $\mathrm{MC}$ simulations in the isothermal-isobaric ensemble containing $N=2000$ particles. Transport properties were determined by MD simulations in the canonical ensemble containing $N=4914$ particles and an integrator time step of $\Delta t=0.88 \mathrm{fs}$. Values were obtained after about $9 \cdot 10^{8}$ time steps, which represents a sampling time of $0.8 \mu \mathrm{s}$.

containing $N=4914$ particles, with values being obtained after about $9 \cdot 10^{8}$ time steps, which represents a sampling time of $0.8 \mu \mathrm{s}$.

The selected $g^{e}$ model (fourth order Margules type polynomial) was fitted with a standard nonlinear least squares algorithm to minimize the root mean square error between simulation data and the model around the equilibrium vapor phase composition $\boldsymbol{y}^{*}=(0.13,0.03,0.84)$ and yielded for the parameters $A_{i j}, E_{i j}$ and $C_{i}$

$$
\begin{aligned}
{\left[\begin{array}{ccc}
- & A_{12} & A_{13} \\
A_{21} & - & A_{23} \\
A_{31} & A_{32} & -
\end{array}\right] } & =\left[\begin{array}{ccc}
- & -39.9 & 1.7 \\
4.2 & - & 3.3 \\
3.8 & 0.7 & -
\end{array}\right] \\
{\left[\begin{array}{ccc}
- & E_{12} & E_{13} \\
- & - & E_{23} \\
- & - & -
\end{array}\right] } & =\left[\begin{array}{ccc}
- & -0.01 & 1.63 \\
- & - & -1.82 \\
- & - & -
\end{array}\right], \\
{\left[\begin{array}{l}
C_{1} \\
C_{2} \\
C_{3}
\end{array}\right] } & =\left[\begin{array}{c}
-50.6 \\
-126.3 \\
-40.9
\end{array}\right]
\end{aligned}
$$

To describe the liquid phase's non-idealities with greater precision, an additional fit around $\boldsymbol{x}^{*}=(0.495,0.505,0)$ was performed to yield different parameters for the same

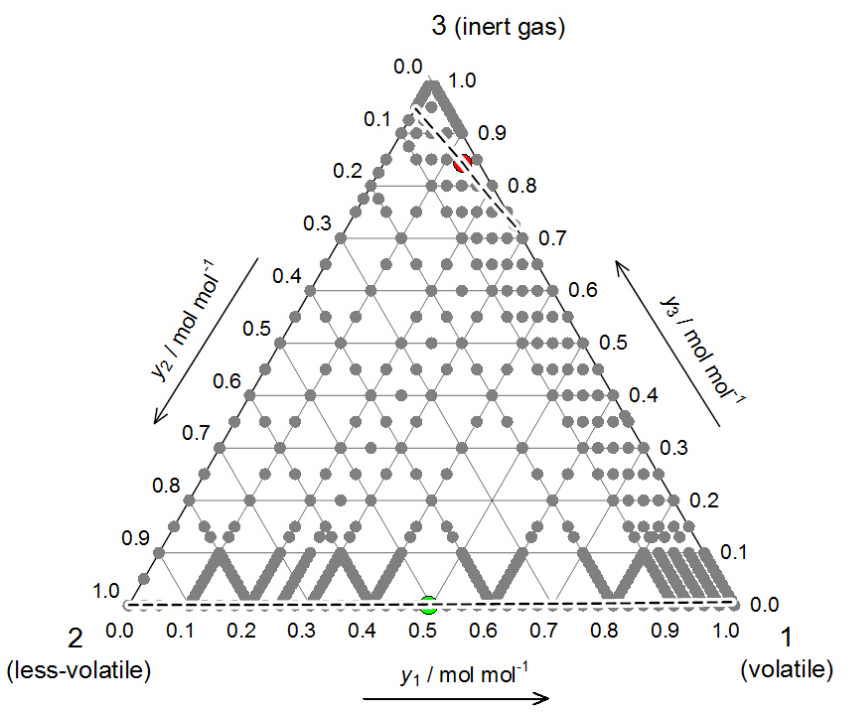

FIG. 1. Gibbs ternary plot illustrating compositions where thermodynamic data were generated by MC simulations. Under equilibrium, the vapor phase's composition is given as $\boldsymbol{y}=(0.13,0.027,0.843) \mathrm{mol} \mathrm{mol}^{-1}$ (red bullet) while the liquid phase's is given as $\boldsymbol{x}=(0.495,0.505,0) \mathrm{mol} \mathrm{mol}^{-1}$ (green bullet). The dashed lines show the vapor and liquid binodals.

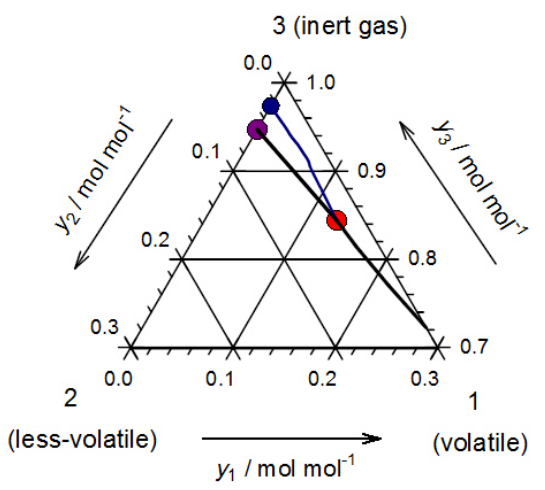

FIG. 2. Segment of the Gibbs ternary plot depicting different paths for the vapor phase composition during evaporation from simulation (blue) and analytic solution (violet).

model applied to the binary subsystem only

$$
\left[\begin{array}{c}
A_{21} \\
A_{12} \\
E_{12}
\end{array}\right]=\left[\begin{array}{c}
0.26 \\
0.25 \\
-0.01
\end{array}\right]
$$

\footnotetext{
a) jadran.vrabec@upb.de
} 


\section{Vapor phase convective motion}

The linear momentum of a multicomponent mixture is constructed out of the vector sum of its constituent's linear momenta

$$
\rho_{v} u_{z}=\sum_{i=1}^{3} \rho_{u, i} v_{z, i} .
$$

The $i$-th component's partial density is given by $\rho_{v, i}=$ $y_{i} \rho_{v}$. The third component is seen as a stagnant gas $u_{z, 3} \approx 0$, i.e. no collective motion in a spatial direction is measurable, although random thermal motion is present. The molar averaged velocity $u_{z}$ in the relevant $z$ direction is then given as a proportionate sum of every component's convective velocity $v_{z, i}$

$$
u_{z}=\sum_{i=1}^{3} y_{i} u_{z, i} \approx \sum_{i=1}^{2} y_{i} u_{z, i} .
$$

The convective motion $u_{z, i}$ can be decomposed into an advective and a diffusive contribution $u_{z, i}=u_{z}+U_{z, i}$

$$
\sum_{i=1}^{2} y_{i} u_{z, i}=\sum_{i=1}^{2} y_{i}\left(u_{z}+U_{z, i}\right) .
$$

Substituting the molar averaged motion $u_{z}$ with the result already obtained from (6) yields

$$
\sum_{i=1}^{2} y_{i} u_{z, i}=\sum_{i=1}^{2} y_{i}\left(\sum_{i=1}^{2} y_{i} u_{z, i}+U_{z, i}\right),
$$

which after a simple algebraic manipulation connects each component's convective $y_{i} v_{z, i}$ to its diffusion flux $y_{i} U_{z, i}$

$$
\sum_{i=1}^{2} y_{i} u_{z, i}\left(1-\sum_{i=1}^{2} y_{i}\right)=\sum_{i=1}^{2} y_{i} U_{z, i}
$$

In a last step, the mixture's total molar composition constraint $y_{1}+y_{2}+y_{3}=1$ is utilized to retain the description used in equation (11) of the manuscript

$$
\sum_{i=1}^{2} y_{i} u_{z, i}=\sum_{i=1}^{2}\left(\frac{y_{i}}{y_{3}}\right) U_{z, i}=\sum_{i=1}^{2} b_{i 3} U_{z, i} .
$$

\section{Boundary condition}

In equation (18) of the manuscript, a boundary condition was used that needs a more detailed clarification concerning its limitations. Akin to the particle density conservation equation

$$
\partial_{t}\left(\rho_{v, i}(z, t)\right)+\partial_{z}\left(\rho_{v, i}(z, t) v_{z, i}(z, t)\right)=0,
$$

a conservation equation for the weighted mole fractions $b_{i 3}$ can be constructed

$$
\partial_{t}\left(b_{i 3}(z)\right)+\partial_{z}\left(b_{i 3}(z) v_{z, i}(z, t)\right)=0,
$$

where utilizing assumption (12) of the manuscript reduces the complexity significantly. It should be noted that the parameters $b_{i 3}$ are indeed functions of time, yet their variation in time was considered negligible. A formulation for this derivative at the interphase $z=z_{s}$ needs to be found

$$
\left.\partial_{z}\left(b_{i 3}(z) v_{z, i}(z, t)\right)\right|_{z_{s}}=0 .
$$

Decomposing the convective velocity vector $v_{z, i}=u_{z}+$ $U_{z, i}$ into its advective and diffusive parts again yields

$$
\left.\partial_{z}\left(b_{i 3}(z)\left(u_{z}+U_{z, i}\right)\right)\right|_{z_{s}}=0 .
$$

The diffusion flux has already been identified to be described by Fick's diffusion law that with the effective diffusion coefficient $\mathcal{D}_{i}$ reads

$$
b_{i 3} U_{z_{i}}=-\mathcal{D}_{i} d_{z}\left(b_{i 3}(z)\right) \text {, }
$$

after neglecting $\left.b_{i 3} d_{z}\left(u_{z}\right)\right|_{z_{s}}$ for being small compared to the other terms

$$
\left.u_{z} d_{z}\left(b_{i 3}(z)\right)\right|_{z_{s}}-\left.\mathcal{D}_{i} d_{z z}\left(b_{i 3}(z)\right)\right|_{z_{s}}=0
$$

In a last step, the connection between this vapor phase argumentation and the liquid phase's surface regression velocity needs to be found. The latter is identified as the negative convective motion of evaporate at the interphase and is given

$$
u_{z, l i q}\left(z_{s}\right)=-u_{z}\left(z_{s}\right)=\left.\sum_{i=1}^{2} \mathcal{D}_{i} d_{z}\left(b_{i 3}(z)\right)\right|_{z_{s}} .
$$

After inserting (17) into (16) and expanding the sum, a further assumption has to be made

$$
\begin{aligned}
& \left.\left(\left.\mathcal{D}_{1} d_{z}\left(b_{13}(z)\right)\right|_{z_{s}}+\left.\mathcal{D}_{2} d_{z}\left(b_{23}(z)\right)\right|_{z_{s}}\right) d_{z}\left(b_{i 3}(z)\right)\right|_{z_{s}} \\
& -\left.\mathcal{D}_{i} d_{z z}\left(b_{i 3}(z)\right)\right|_{z_{s}}=0 .
\end{aligned}
$$

All crossover terms are assumed to be negligible over the non-crossover terms

$\left.\left.\mathcal{D}_{j} d_{z}\left(b_{j 3}(z)\right)\right|_{z_{s}} d_{z}\left(b_{i 3}(z)\right)\right|_{z_{s}} \ll \mathcal{D}_{i}\left(\left.d_{z}\left(b_{i 3}(z)\right)\right|_{z_{s}}\right)^{2}, \forall j \neq i$.

One can finally conclude the problem's boundary condition at film's surface to be

$$
\left(\left.d_{z}\left(b_{i 3}(z)\right)\right|_{z_{s}}\right)^{2}=\left.d_{z z}\left(b_{i 3}(z)\right)\right|_{z_{s}} .
$$


TABLE I. Pure component simulation results that give the behavior of each component's chemical potential $\mu_{0, i}$ and residual contribution $\psi_{i}$ as a function of pressure at constant temperature $T=80 \mathrm{~K}$. The simulations yielded a uniform molar density of $\rho=0.0151 \mathrm{~mol} \mathrm{dm}^{-3}$ for each component prepared in the state $T=80 \mathrm{~K}, p=0.01 \mathrm{MPa}$ that is precisely the ideal gas equation's result and determines the ideal gas chemical potentials to be $\boldsymbol{\mu}_{0}^{0}=-(7.86,7.86,7.85)$. Since the residual contribution $\psi_{i}$, according to (27), measures a component's departure from the ideal gas behavior, the components 1 and 2 are liquid while component 3 is gaseous for the system being prepared in the state $T=80 \mathrm{~K}, p=6.34 \mathrm{MPa}$.

\begin{tabular}{cccccc}
\hline \hline$p / \mathrm{MPa}$ & $\mu_{0,1}$ & $\mu_{0,2}$ & $\mu_{0,3}$ & $\psi_{1}$ & $\psi_{2}$ \\
\hline 0.01 & -7.86 & -7.86 & -7.85 & 1 & 1 \\
0.10 & -5.56 & -5.57 & -5.55 & 0.990 & 0.983 \\
1.00 & -3.37 & -4.80 & -3.23 & 0.885 & 0.247 \\
2.00 & -3.05 & -4.68 & -2.51 & 0.764 & 0.120 \\
3.00 & -3.01 & -4.65 & -2.09 & 0.425 & 0.082 \\
3.25 & -3.00 & & -2.00 & 0.397 & 1.021 \\
3.50 & -2.99 & & -1.92 & 0.373 & 1.067 \\
3.75 & -2.98 & -1.85 & 0.350 & 1.073 \\
4.00 & -2.97 & 4.62 & -1.78 & 0.332 & 1.079 \\
4.25 & -2.96 & & -1.71 & 0.315 & 1.085 \\
4.50 & -2.95 & -1.65 & 0.300 & 1.091 \\
4.75 & -2.94 & -1.59 & 0.287 & 1.097 \\
6.00 & -2.93 & -1.53 & 0.275 & 1.103 \\
5.25 & -2.92 & -4.59 & -1.48 & 0.265 & 1.109 \\
5.50 & -2.91 & -1.42 & 0.255 & 1.115 \\
5.75 & -2.91 & -1.37 & 0.246 & 1.122 \\
6.00 & -2.90 & -2.89 & -1.33 & 0.238 & 1.128 \\
6.25 & -2.89 & -4.56 & -1.28 & 0.230 & 1.134 \\
6.34 & & & 0.228 & 1.141 \\
\hline \hline
\end{tabular}

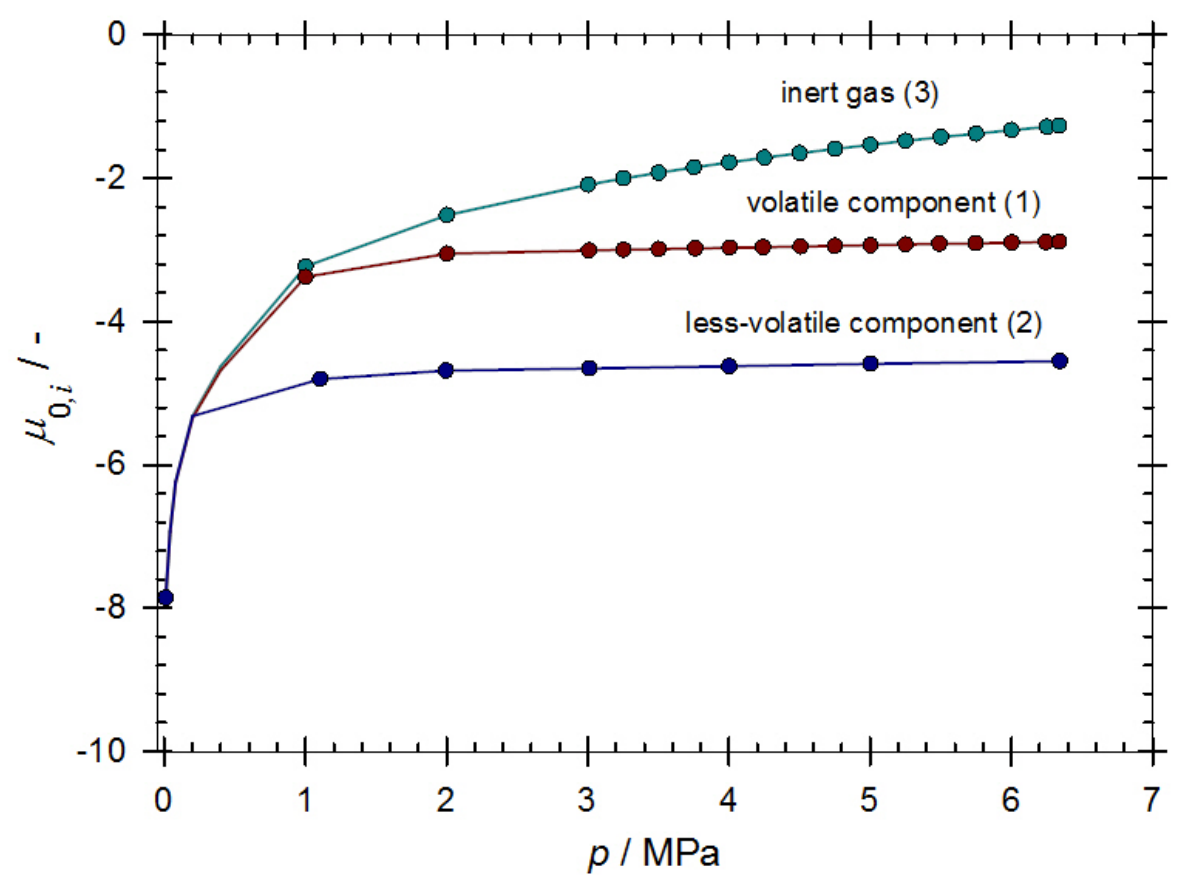

FIG. 3. Graphical representation of the individual pure component's chemical potentials $\mu_{0, i}$ as a function of pressure at constant temperature. The three curves, as they should, approach identical values for the ideal gas case. 
TABLE II. The binary subsystem that is composed of volatile (component 1) and less-volatile (component 2) particles remains liquid for all possible compositions. The fourth order Margules type polynomial covers also the respective infinite dilution activity coefficients $\gamma_{i}^{\infty}$, as depicted in Fiq.(4).

\begin{tabular}{|c|c|c|c|c|c|c|c|c|}
\hline$x_{1}$ & $x_{2}$ & $y_{3}$ & $g_{s i m}^{e}$ & $g_{\text {mod }}^{e}$ & $\gamma_{1, \text { sim }}$ & $\gamma_{2, \text { sim }}$ & $\gamma_{1, \text { mod }}$ & $\gamma_{2, \bmod }$ \\
\hline 0 & 1 & 0 & 0 & 0 & - & 1 & 1.285 & 1 \\
\hline 0.025 & 0.975 & 0 & 0.01 & 0.01 & 1.269 & 1.004 & 1.270 & 1.000 \\
\hline 0.050 & 0.950 & 0 & 0.02 & 0.01 & 1.258 & 1.007 & 1.256 & 1.001 \\
\hline 0.075 & 0.925 & 0 & 0.02 & 0.02 & 1.238 & 1.001 & 1.242 & 1.001 \\
\hline 0.100 & 0.900 & 0 & 0.02 & 0.02 & 1.222 & 1.001 & 1.228 & 1.002 \\
\hline 0.125 & 0.875 & 0 & 0.02 & 0.03 & 1.204 & 0.998 & 1.215 & 1.004 \\
\hline 0.150 & 0.850 & 0 & 0.03 & 0.03 & 1.194 & 1.003 & 1.202 & 1.005 \\
\hline 0.175 & 0.825 & 0 & 0.04 & 0.04 & 1.194 & 1.014 & 1.190 & 1.007 \\
\hline 0.200 & 0.800 & 0 & 0.03 & 0.04 & 1.168 & 1.003 & 1.178 & 1.010 \\
\hline 0.225 & 0.775 & 0 & 0.05 & 0.04 & 1.116 & 1.014 & 1.166 & 1.012 \\
\hline 0.250 & 0.750 & 0 & 0.05 & 0.05 & 1.158 & 1.021 & 1.155 & 1.016 \\
\hline 0.275 & 0.725 & 0 & 0.05 & 0.05 & 1.144 & 1.020 & 1.145 & 1.019 \\
\hline 0.300 & 0.700 & 0 & 0.06 & 0.05 & 1.137 & 1.027 & 1.135 & 1.023 \\
\hline 0.325 & 0.675 & 0 & 0.05 & 0.06 & 1.120 & 1.023 & 1.125 & 1.027 \\
\hline 0.350 & 0.650 & 0 & 0.06 & 0.06 & 1.118 & 1.034 & 1.115 & 1.031 \\
\hline 0.375 & 0.625 & 0 & 0.06 & 0.06 & 1.104 & 1.032 & 1.106 & 1.036 \\
\hline 0.400 & 0.600 & 0 & 0.06 & 0.06 & 1.099 & 1.043 & 1.098 & 1.041 \\
\hline 0.425 & 0.575 & 0 & 0.06 & 0.06 & 1.089 & 1.045 & 1.090 & 1.046 \\
\hline 0.450 & 0.550 & 0 & 0.06 & 0.06 & 1.081 & 1.050 & 1.082 & 1.052 \\
\hline 0.475 & 0.525 & 0 & 0.06 & 0.06 & 1.075 & 1.058 & 1.074 & 1.059 \\
\hline 0.495 & 0.505 & 0 & 0.06 & 0.06 & 1.070 & 1.061 & 1.069 & 1.064 \\
\hline 0.525 & 0.475 & 0 & 0.06 & 0.06 & 1.061 & 1.071 & 1.061 & 1.072 \\
\hline 0.550 & 0.450 & 0 & 0.06 & 0.06 & 1.056 & 1.079 & 1.054 & 1.080 \\
\hline 0.575 & 0.425 & 0 & 0.07 & 0.06 & 1.052 & 1.090 & 1.048 & 1.088 \\
\hline 0.600 & 0.400 & 0 & 0.06 & 0.06 & 1.041 & 1.091 & 1.043 & 1.096 \\
\hline 0.625 & 0.375 & 0 & 0.06 & 0.06 & 1.041 & 1.105 & 1.037 & 1.105 \\
\hline 0.650 & 0.350 & 0 & 0.06 & 0.06 & 1.034 & 1.113 & 1.033 & 1.114 \\
\hline 0.675 & 0.325 & 0 & 0.06 & 0.06 & 1.029 & 1.123 & 1.028 & 1.124 \\
\hline 0.700 & 0.300 & 0 & 0.05 & 0.05 & 1.022 & 1.128 & 1.024 & 1.134 \\
\hline 0.725 & 0.275 & 0 & 0.05 & 0.05 & 1.020 & 1.142 & 1.020 & 1.144 \\
\hline 0.750 & 0.250 & 0 & 0.05 & 0.05 & 1.017 & 1.153 & 1.016 & 1.155 \\
\hline 0.775 & 0.225 & 0 & 0.04 & 0.05 & 1.013 & 1.163 & 1.013 & 1.167 \\
\hline 0.800 & 0.200 & 0 & 0.04 & 0.04 & 1.012 & 1.179 & 1.011 & 1.179 \\
\hline 0.825 & 0.175 & 0 & 0.04 & 0.04 & 1.009 & 1.192 & 1.008 & 1.192 \\
\hline 0.850 & 0.150 & 0 & 0.03 & 0.03 & 1.005 & 1.203 & 1.006 & 1.205 \\
\hline 0.875 & 0.125 & 0 & 0.03 & 0.03 & 1.004 & 1.218 & 1.004 & 1.218 \\
\hline 0.900 & 0.100 & 0 & 0.02 & 0.02 & 1.003 & 1.233 & 1.003 & 1.233 \\
\hline 0.925 & 0.075 & 0 & 0.02 & 0.02 & 1.002 & 1.250 & 1.001 & 1.247 \\
\hline 0.950 & 0.050 & 0 & 0.01 & 0.01 & 1.001 & 1.266 & 1.001 & 1.263 \\
\hline 0.975 & 0.025 & 0 & 0.01 & 0.01 & 1.000 & 1.282 & 1.000 & 1.279 \\
\hline 1 & 0 & 0 & 0 & 0 & 1 & - & 1 & 1.295 \\
\hline
\end{tabular}


TABLE III. Data for the binary subsystem that is composed of volatile (component 1) and inert gas (component 3) particles. Thermodynamic stability analysis relates the thermodynamic factor's algebraic sign to either a stable $(\Gamma>0)$ or an unstable $(\Gamma<0)$ state. This binary mixture must exhibit a vapor-liquid phase separation between $0.45<y_{1}<0.92$. Both the Gibbs excess energy and the fugacity coefficient $\vartheta_{1}$ are well described in the vapor phase by the selected $g^{e}$ model.

\begin{tabular}{|c|c|c|c|c|c|c|c|}
\hline$y_{1}$ & $y_{2}$ & $y_{3}$ & $g_{s i m}^{e}$ & $g_{\text {mod }}^{e}$ & $\vartheta_{1, \text { sim }}$ & $\vartheta_{1, \bmod }$ & $\Gamma_{1}$ \\
\hline 0 & 0 & 1 & 0 & 0 & - & 5 & 1 \\
\hline 0.010 & 0 & 0.990 & 0.02 & 0.02 & 5.10 & 5.16 & 0.98 \\
\hline 0.020 & 0 & 0.980 & 0.03 & 0.03 & 5.02 & 5.07 & 0.96 \\
\hline 0.025 & 0 & 0.975 & 0.04 & 0.04 & 4.98 & 5.03 & 0.96 \\
\hline 0.030 & 0 & 0.970 & 0.05 & 0.05 & 4.94 & 4.98 & 0.95 \\
\hline 0.040 & 0 & 0.960 & 0.06 & 0.06 & 4.87 & 4.90 & 0.93 \\
\hline 0.050 & 0 & 0.950 & 0.08 & 0.08 & 4.79 & 4.82 & 0.92 \\
\hline 0.060 & 0 & 0.940 & 0.10 & 0.10 & 4.72 & 4.74 & 0.91 \\
\hline 0.070 & 0 & 0.930 & 0.11 & 0.11 & 4.65 & 4.67 & 0.89 \\
\hline 0.080 & 0 & 0.920 & 0.13 & 0.13 & 4.59 & 4.60 & 0.88 \\
\hline 0.090 & 0 & 0.910 & 0.14 & 0.14 & 4.52 & 4.54 & 0.87 \\
\hline 0.100 & 0 & 0.900 & 0.16 & 0.16 & 4.45 & 4.47 & 0.86 \\
\hline 0.150 & 0 & 0.850 & 0.23 & 0.23 & 4.15 & 4.18 & 0.81 \\
\hline 0.200 & 0 & 0.800 & 0.30 & 0.30 & 3.88 & 3.93 & 0.75 \\
\hline 0.250 & 0 & 0.750 & 0.36 & 0.36 & 3.63 & 3.68 & 0.67 \\
\hline 0.275 & 0 & 0.725 & 0.39 & 0.39 & 3.52 & 3.56 & 0.63 \\
\hline 0.300 & 0 & 0.700 & 0.42 & 0.42 & 3.40 & 3.44 & 0.58 \\
\hline 0.350 & 0 & 0.650 & 0.48 & 0.48 & 3.20 & 3.20 & 0.46 \\
\hline 0.400 & 0 & 0.600 & 0.53 & 0.53 & 3.00 & 2.95 & 0.32 \\
\hline 0.450 & 0 & 0.550 & 0.57 & 0.57 & 2.81 & 2.70 & 0.16 \\
\hline 0.500 & 0 & 0.500 & 0.61 & 0.60 & 2.63 & 2.45 & -0.01 \\
\hline 0.550 & 0 & 0.450 & 0.63 & 0.63 & 2.31 & 2.21 & -0.18 \\
\hline 0.600 & 0 & 0.400 & 0.64 & 0.64 & 2.01 & 1.98 & -0.35 \\
\hline 0.639 & 0 & 0.362 & 0.64 & 0.64 & 1.86 & 1.81 & -0.46 \\
\hline 0.650 & 0 & 0.350 & 0.63 & 0.64 & 1.83 & 1.76 & -0.49 \\
\hline 0.700 & 0 & 0.300 & 0.62 & 0.62 & 1.64 & 1.57 & -0.60 \\
\hline 0.750 & 0 & 0.250 & 0.59 & 0.58 & 1.50 & 1.41 & -0.64 \\
\hline 0.800 & 0 & 0.200 & 0.54 & 0.52 & 1.33 & 1.27 & -0.60 \\
\hline 0.850 & 0 & 0.150 & 0.46 & 0.44 & 1.23 & 1.15 & -0.44 \\
\hline 0.900 & 0 & 0.100 & 0.37 & 0.32 & 1.12 & 1.07 & -0.15 \\
\hline 0.910 & 0 & 0.090 & 0.35 & 0.30 & 1.10 & 1.06 & -0.07 \\
\hline 0.920 & 0 & 0.080 & 0.32 & 0.27 & 1.08 & 1.05 & 0.02 \\
\hline 0.930 & 0 & 0.070 & 0.29 & 0.24 & 1.06 & 1.04 & 0.11 \\
\hline 0.940 & 0 & 0.060 & 0.26 & 0.21 & 1.04 & 1.03 & 0.21 \\
\hline 0.950 & 0 & 0.050 & 0.23 & 0.18 & 1.02 & 1.02 & 0.32 \\
\hline 0.960 & 0 & 0.040 & 0.18 & 0.15 & 1.01 & 1.01 & 0.44 \\
\hline 0.970 & 0 & 0.030 & 0.14 & 0.11 & 1.01 & 1.01 & 0.57 \\
\hline 0.980 & 0 & 0.020 & 0.09 & 0.08 & 1.00 & 1.00 & 0.70 \\
\hline 0.990 & 0 & 0.010 & 0.05 & 0.04 & 1.00 & 1.00 & 0.85 \\
\hline 1 & 0 & 0 & 0 & 0 & 1 & 1 & 1 \\
\hline
\end{tabular}


TABLE IV. Data for the binary subsystem that is composed of less-volatile and inert gas particles. This subsystem exhibits a wider phase transition in terms of molar variation than the system in table (III). This subsystem must exhibit a vapor-liquid phase separation in the range $0.175<y_{2}<0.8$.

\begin{tabular}{|c|c|c|c|c|c|c|c|}
\hline$y_{1}$ & $y_{2}$ & $y_{3}$ & $g_{\text {sim }}^{e}$ & $g_{\text {mod }}^{e}$ & $\vartheta_{2, s i m}$ & $\vartheta_{2, \text { mod }}$ & $\Gamma_{2}$ \\
\hline 0 & 0 & 1 & 0 & 0 & - & 35.34 & 1 \\
\hline 0 & 0.010 & 0.990 & 0.03 & 0.04 & 26.33 & 32.93 & 0.95 \\
\hline 0 & 0.020 & 0.980 & 0.07 & 0.07 & 25.71 & 30.74 & 0.90 \\
\hline 0 & 0.031 & 0.969 & 0.10 & 0.11 & 25.06 & 28.55 & 0.83 \\
\hline 0 & 0.040 & 0.960 & 0.13 & 0.14 & 24.54 & 26.91 & 0.78 \\
\hline 0 & 0.043 & 0.957 & 0.14 & 0.15 & 24.31 & 26.50 & 0.77 \\
\hline 0 & 0.050 & 0.950 & 0.16 & 0.17 & 23.98 & 25.23 & 0.72 \\
\hline 0 & 0.100 & 0.900 & 0.32 & 0.33 & 21.40 & 18.69 & 0.41 \\
\hline 0 & 0.150 & 0.850 & 0.47 & 0.47 & 19.13 & 14.28 & 0.10 \\
\hline 0 & 0.175 & 0.825 & 0.54 & 0.53 & 18.07 & 12.62 & -0.06 \\
\hline 0 & 0.200 & 0.800 & 0.61 & 0.59 & 16.61 & 11.23 & -0.21 \\
\hline 0 & 0.255 & 0.775 & 0.62 & 0.65 & 9.23 & 10.05 & -0.36 \\
\hline 0 & 0.250 & 0.750 & 0.66 & 0.70 & 7.65 & 9.04 & -0.49 \\
\hline 0 & 0.300 & 0.700 & 0.74 & 0.78 & 6.09 & 7.42 & -0.73 \\
\hline 0 & 0.350 & 0.650 & 0.81 & 0.85 & 5.27 & 6.20 & -0.91 \\
\hline 0 & 0.400 & 0.600 & 0.87 & 0.89 & 4.53 & 5.25 & -1.02 \\
\hline 0 & 0.450 & 0.550 & 0.90 & 0.91 & 3.88 & 4.50 & -1.07 \\
\hline 0 & 0.500 & 0.500 & 0.93 & 0.91 & 3.40 & 3.89 & -1.05 \\
\hline 0 & 0.550 & 0.450 & 0.94 & 0.89 & 2.95 & 3.38 & -0.96 \\
\hline 0 & 0.600 & 0.400 & 0.93 & 0.85 & 2.56 & 2.95 & -0.81 \\
\hline 0 & 0.650 & 0.350 & 0.91 & 0.79 & 2.19 & 2.58 & -0.60 \\
\hline 0 & 0.700 & 0.300 & 0.88 & 0.72 & 1.89 & 2.26 & -0.35 \\
\hline 0 & 0.750 & 0.250 & 0.82 & 0.63 & 1.63 & 1.98 & -0.07 \\
\hline 0 & 0.800 & 0.200 & 0.75 & 0.52 & 1.44 & 1.73 & 0.22 \\
\hline 0 & 0.850 & 0.150 & 0.64 & 0.40 & 1.30 & 1.51 & 0.49 \\
\hline 0 & 0.900 & 0.100 & 0.51 & 0.28 & 1.13 & 1.32 & 0.74 \\
\hline 0 & 0.950 & 0.005 & 0.31 & 0.14 & 1.04 & 1.15 & 0.92 \\
\hline 0 & 1 & 0 & 0 & 0 & 1 & 1 & 1 \\
\hline
\end{tabular}

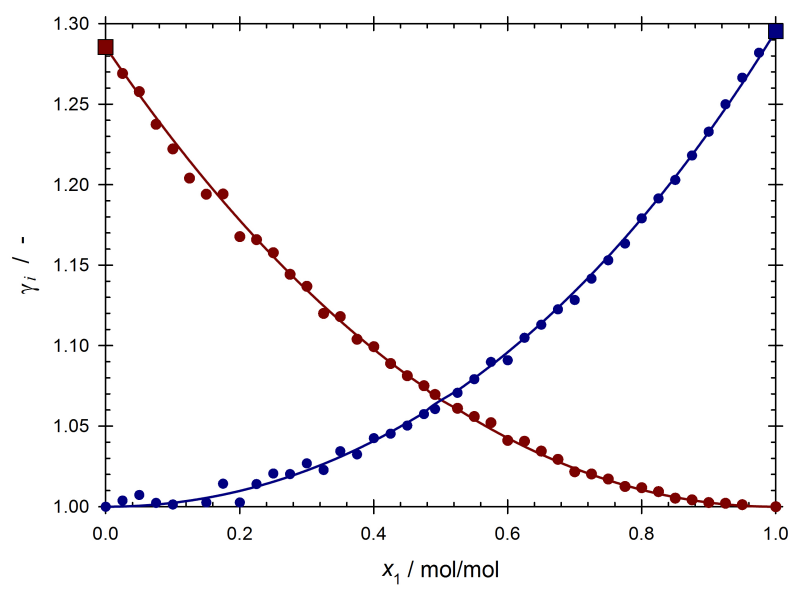

FIG. 4. Binary liquid mixture's (components 1 and 2) activity coefficients as given in table (III). The lines represent the Margules polynomial model while the bullets represent simulation data. The activity coefficients at infinite dilution $\gamma_{i}^{\infty}$ (squares) are well covered by the chosen Margules type polynomial. 
TABLE V. Data for the ternary system, where the first component's mole fraction is varied and the second's is kept constant at $y_{2}=0.025 \mathrm{~mol} \mathrm{~mol}^{-1}$.

\begin{tabular}{|c|c|c|c|c|c|c|c|c|c|c|c|c|}
\hline$y_{1}$ & $y_{2}$ & $y_{3}$ & $g_{\text {sim }}^{e}$ & $g_{\text {mod }}^{e}$ & $\vartheta_{1, \text { sim }}$ & $\vartheta_{2, s i m}$ & $\vartheta_{1, \bmod }$ & $\vartheta_{2, \bmod }$ & $\Gamma_{11}$ & $\Gamma_{12}$ & $\Gamma_{21}$ & $\Gamma_{22}$ \\
\hline 0 & 0.025 & 0.975 & 0.08 & 0.08 & - & 25.41 & 4.96 & 25.48 & 1 & 0 & -0.06 & 0.95 \\
\hline 0.025 & 0.025 & 0.950 & 0.12 & 0.12 & 4.75 & 24.25 & 4.74 & 24.25 & 0.95 & -0.05 & -0.05 & 0.98 \\
\hline 0.050 & 0.025 & 0.925 & 0.16 & 0.16 & 4.58 & 23.16 & 4.55 & 23.22 & 0.92 & -0.09 & -0.04 & 1.00 \\
\hline 0.070 & 0.025 & 0.905 & 0.19 & 0.20 & 4.45 & 22.33 & 4.41 & 22.51 & 0.89 & -0.11 & -0.04 & 1.01 \\
\hline 0.075 & 0.025 & 0.900 & 0.20 & 0.20 & 4.42 & 22.13 & 4.38 & 22.34 & 0.88 & -0.11 & -0.04 & 1.01 \\
\hline 0.100 & 0.025 & 0.875 & 0.23 & 0.24 & 4.26 & 21.16 & 4.23 & 21.58 & 0.85 & -0.15 & -0.04 & 1.01 \\
\hline 0.110 & 0.025 & 0.865 & 0.25 & 0.26 & 4.20 & 20.79 & 4.17 & 21.29 & 0.84 & -0.16 & -0.03 & 1.01 \\
\hline 0.120 & 0.025 & 0.855 & 0.26 & 0.27 & 4.15 & 20.42 & 4.11 & 21.02 & 0.83 & -0.17 & -0.03 & 1.01 \\
\hline 0.125 & 0.025 & 0.850 & 0.27 & 0.28 & 4.12 & 20.24 & 4.09 & 20.88 & 0.82 & -0.18 & -0.03 & 1.00 \\
\hline 0.130 & 0.025 & 0.845 & 0.28 & 0.29 & 4.09 & 20.07 & 4.06 & 20.75 & 0.82 & -0.18 & -0.03 & 1.00 \\
\hline 0.140 & 0.025 & 0.835 & 0.29 & 0.30 & 4.03 & 19.72 & 4.01 & 20.49 & 0.81 & -0.19 & -0.03 & 0.99 \\
\hline 0.150 & 0.025 & 0.825 & 0.30 & 0.31 & 3.98 & 19.38 & 3.96 & 20.24 & 0.79 & -0.20 & -0.03 & 0.98 \\
\hline 0.160 & 0.025 & 0.815 & 0.32 & 0.33 & 3.93 & 19.04 & 3.91 & 19.99 & 0.78 & -0.21 & -0.03 & 0.97 \\
\hline 0.170 & 0.025 & 0.805 & 0.33 & 0.34 & 3.87 & 18.71 & 3.86 & 19.74 & 0.77 & -0.23 & -0.03 & 0.96 \\
\hline 0.175 & 0.025 & 0.800 & 0.34 & 0.35 & 3.85 & 18.55 & 3.83 & 19.62 & 0.76 & -0.23 & -0.03 & 0.95 \\
\hline 0.180 & 0.025 & 0.795 & 0.34 & 0.36 & 3.82 & 18.39 & 3.81 & 19.49 & 0.76 & -0.24 & -0.03 & 0.94 \\
\hline 0.190 & 0.025 & 0.785 & 0.36 & 0.37 & 3.77 & 18.08 & 3.76 & 19.24 & 0.74 & -0.25 & -0.03 & 0.92 \\
\hline 0.200 & 0.025 & 0.775 & 0.37 & 0.38 & 3.72 & 17.76 & 3.71 & 18.99 & 0.73 & -0.26 & -0.03 & 0.91 \\
\hline 0.225 & 0.025 & 0.750 & 0.40 & 0.42 & 3.60 & 17.01 & 3.59 & 18.36 & 0.69 & -0.30 & -0.04 & 0.85 \\
\hline 0.250 & 0.025 & 0.725 & 0.43 & 0.45 & 3.49 & 16.29 & 3.48 & 17.69 & 0.65 & -0.35 & -0.04 & 0.79 \\
\hline 0.270 & 0.025 & 0.705 & 0.45 & 0.47 & 3.40 & 15.74 & 3.39 & 17.13 & 0.62 & -0.38 & -0.04 & 0.73 \\
\hline 0.275 & 0.025 & 0.700 & 0.46 & 0.48 & 3.38 & 15.60 & 3.37 & 16.98 & 0.61 & -0.39 & -0.04 & 0.71 \\
\hline 0.300 & 0.025 & 0.675 & 0.49 & 0.51 & 3.27 & 14.94 & 3.25 & 16.23 & 0.56 & -0.45 & -0.05 & 0.63 \\
\hline 0.310 & 0.025 & 0.665 & 0.50 & 0.52 & 3.23 & 14.68 & 3.21 & 15.91 & 0.54 & -0.47 & -0.05 & 0.59 \\
\hline 0.320 & 0.025 & 0.655 & 0.51 & 0.53 & 3.19 & 14.42 & 3.16 & 15.59 & 0.52 & -0.49 & -0.05 & 0.55 \\
\hline 0.325 & 0.025 & 0.650 & 0.51 & 0.53 & 3.17 & 14.29 & 3.14 & 15.42 & 0.51 & -0.51 & -0.06 & 0.54 \\
\hline 0.340 & 0.025 & 0.635 & 0.54 & 0.55 & 3.11 & 13.91 & 3.07 & 14.91 & 0.47 & -0.54 & -0.06 & 0.47 \\
\hline 0.350 & 0.025 & 0.625 & 0.55 & 0.56 & 3.07 & 13.66 & 3.02 & 14.56 & 0.45 & -0.57 & -0.06 & 0.43 \\
\hline 0.360 & 0.025 & 0.615 & 0.56 & 0.57 & 3.03 & 13.42 & 2.98 & 14.21 & 0.42 & -0.60 & -0.06 & 0.39 \\
\hline 0.370 & 0.025 & 0.605 & 0.56 & 0.58 & 2.99 & 13.17 & 2.93 & 13.84 & 0.40 & -0.60 & -0.07 & 0.34 \\
\hline 0.380 & 0.025 & 0.595 & 0.58 & 0.59 & 2.95 & 12.93 & 2.88 & 13.47 & 0.37 & -0.65 & -0.07 & 0.29 \\
\hline 0.390 & 0.025 & 0.585 & 0.59 & 0.60 & 2.92 & 12.69 & 2.84 & 13.09 & 0.34 & -0.68 & -0.07 & 0.24 \\
\hline 0.400 & 0.025 & 0.575 & 0.61 & 0.60 & 2.88 & 12.45 & 2.79 & 12.71 & 0.32 & -0.71 & -0.08 & 0.19 \\
\hline 0.425 & 0.025 & 0.550 & 0.64 & 0.62 & 2.79 & 11.85 & 2.67 & 11.73 & 0.25 & -0.77 & -0.09 & 0.06 \\
\hline 0.475 & 0.025 & 0.500 & 0.65 & 0.66 & 2.59 & 10.44 & 2.44 & 9.72 & 0.10 & -0.90 & -0.10 & -0.24 \\
\hline 0.525 & 0.025 & 0.450 & 0.66 & 0.68 & 2.16 & 6.10 & 2.22 & 7.74 & -0.06 & -1.00 & -0.12 & -0.58 \\
\hline 0.575 & 0.025 & 0.400 & 0.65 & 0.69 & 1.96 & 4.94 & 2.00 & 5.91 & -0.20 & -1.03 & -0.15 & -0.95 \\
\hline 0.675 & 0.025 & 0.300 & 0.63 & 0.67 & 1.63 & 3.50 & 1.62 & 2.99 & -0.40 & -0.77 & -0.19 & -1.81 \\
\hline 0.725 & 0.025 & 0.250 & 0.60 & 0.63 & 1.48 & 2.93 & 1.47 & 1.98 & -0.42 & -0.40 & -0.22 & -2.29 \\
\hline 0.825 & 0.025 & 0.150 & 0.48 & 0.49 & 1.22 & 2.09 & 1.23 & 0.75 & -0.17 & 1.11 & -0.27 & -3.35 \\
\hline 0.875 & 0.025 & 0.100 & 0.38 & 0.37 & 1.11 & 1.76 & 1.16 & 0.43 & 0.14 & 2.36 & -0.29 & -3.94 \\
\hline 0.885 & 0.025 & 0.090 & 0.36 & 0.35 & 1.09 & 1.70 & 1.15 & 0.38 & 0.23 & 2.66 & -0.29 & -4.06 \\
\hline 0.895 & 0.025 & 0.080 & 0.33 & 0.32 & 1.07 & 1.64 & 1.14 & 0.34 & 0.32 & 2.97 & -0.30 & -4.18 \\
\hline 0.905 & 0.025 & 0.070 & 0.30 & 0.29 & 1.05 & 1.59 & 1.13 & 0.30 & 0.42 & 3.31 & -0.30 & -4.30 \\
\hline 0.915 & 0.025 & 0.060 & 0.27 & 0.26 & 1.04 & 1.54 & 1.12 & 0.26 & 0.52 & 3.66 & -0.31 & -4.43 \\
\hline 0.925 & 0.025 & 0.050 & 0.24 & 0.23 & 1.02 & 1.48 & 1.12 & 0.23 & 0.63 & 4.03 & -0.31 & -4.55 \\
\hline 0.935 & 0.025 & 0.040 & 0.19 & 0.20 & 1.01 & 1.44 & 1.11 & 0.21 & 0.76 & 4.42 & -0.32 & -4.68 \\
\hline 0.955 & 0.025 & 0.020 & 0.10 & 0.13 & 1.00 & 1.35 & 1.11 & 0.16 & 1.03 & 5.27 & -0.33 & -4.94 \\
\hline 0.965 & 0.025 & 0.010 & 0.05 & 0.09 & 1.00 & 1.31 & 1.11 & 0.14 & 1.18 & 5.72 & -0.33 & -5.07 \\
\hline 0.975 & 0.025 & 0 & 0.01 & 0.05 & 1.00 & 1.28 & 1.11 & 0.12 & 1.33 & 6.20 & -0.33 & -5.20 \\
\hline
\end{tabular}


TABLE VI. Continuation of table (V) for $y_{2}=0.050 \mathrm{~mol} \mathrm{~mol}^{-1}$.

\begin{tabular}{|c|c|c|c|c|c|c|c|c|c|c|c|c|}
\hline$y_{1}$ & $y_{2}$ & $y_{3}$ & $g_{\text {sim }}^{e}$ & $g_{\text {mod }}^{e}$ & $\vartheta_{1, \mathrm{sim}}$ & $\vartheta_{2, s i m}$ & $\vartheta_{1, \bmod }$ & $\vartheta_{2, \text { mod }}$ & $\Gamma_{11}$ & $\Gamma_{12}$ & $\Gamma_{21}$ & $\Gamma_{22}$ \\
\hline 0 & 0.050 & 0.950 & 0.16 & 0.16 & - & 23.98 & 4.78 & 23.87 & 1 & 0 & -0.04 & 0.84 \\
\hline 0.025 & 0.050 & 0.925 & 0.20 & 0.21 & 4.54 & 22.90 & 4.58 & 23.45 & 0.95 & -0.02 & -0.05 & 0.90 \\
\hline 0.050 & 0.050 & 0.900 & 0.24 & 0.25 & 4.38 & 21.88 & 4.41 & 22.93 & 0.91 & -0.06 & -0.06 & 0.93 \\
\hline 0.070 & 0.050 & 0.880 & 0.27 & 0.28 & 4.26 & 21.11 & 4.28 & 22.42 & 0.88 & -0.07 & -0.07 & 0.93 \\
\hline 0.075 & 0.050 & 0.875 & 0.27 & 0.29 & 4.23 & 20.92 & 4.25 & 22.28 & 0.88 & -0.07 & -0.07 & 0.93 \\
\hline 0.100 & 0.050 & 0.850 & 0.31 & 0.33 & 4.09 & 20.02 & 4.10 & 21.51 & 0.84 & -0.17 & -0.09 & 0.92 \\
\hline 0.110 & 0.050 & 0.840 & 0.32 & 0.34 & 4.03 & 19.67 & 4.05 & 21.16 & 0.83 & -0.20 & -0.09 & 0.91 \\
\hline 0.120 & 0.050 & 0.830 & 0.34 & 0.36 & 3.98 & 19.33 & 3.99 & 20.78 & 0.82 & -0.24 & -0.10 & 0.90 \\
\hline 0.125 & 0.050 & 0.825 & 0.34 & 0.37 & 3.95 & 19.16 & 3.96 & 20.59 & 0.81 & -0.25 & -0.10 & 0.89 \\
\hline 0.130 & 0.050 & 0.820 & 0.35 & 0.37 & 3.92 & 18.99 & 3.94 & 20.39 & 0.80 & -0.27 & -0.11 & 0.88 \\
\hline 0.140 & 0.050 & 0.810 & 0.36 & 0.39 & 3.87 & 18.66 & 3.88 & 19.97 & 0.79 & -0.31 & -0.12 & 0.86 \\
\hline 0.150 & 0.050 & 0.800 & 0.38 & 0.40 & 3.82 & 18.34 & 3.83 & 19.52 & 0.78 & -0.34 & -0.12 & 0.84 \\
\hline 0.160 & 0.050 & 0.790 & 0.39 & 0.42 & 3.77 & 18.03 & 3.78 & 19.06 & 0.76 & -0.38 & -0.13 & 0.82 \\
\hline 0.170 & 0.050 & 0.780 & 0.40 & 0.43 & 3.72 & 17.72 & 3.73 & 18.58 & 0.75 & -0.42 & -0.14 & 0.79 \\
\hline 0.175 & 0.050 & 0.775 & 0.41 & 0.44 & 3.69 & 17.56 & 3.71 & 18.33 & 0.74 & -0.45 & -0.15 & 0.77 \\
\hline 0.180 & 0.050 & 0.770 & 0.41 & 0.44 & 3.67 & 17.41 & 3.68 & 18.07 & 0.73 & -0.47 & -0.15 & 0.75 \\
\hline 0.190 & 0.050 & 0.760 & 0.43 & 0.46 & 3.76 & 16.82 & 3.64 & 17.55 & 0.72 & -0.51 & -0.16 & 0.72 \\
\hline 0.200 & 0.050 & 0.750 & 0.44 & 0.47 & 3.57 & 16.82 & 3.59 & 17.01 & 0.70 & -0.56 & -0.17 & 0.68 \\
\hline 0.225 & 0.050 & 0.725 & 0.47 & 0.50 & 3.36 & 16.10 & 3.47 & 15.60 & 0.66 & -0.69 & -0.19 & 0.57 \\
\hline 0.250 & 0.050 & 0.700 & 0.50 & 0.53 & 3.35 & 15.41 & 3.35 & 14.12 & 0.62 & -0.82 & -0.22 & 0.44 \\
\hline 0.270 & 0.050 & 0.680 & 0.52 & 0.56 & 3.27 & 14.88 & 3.26 & 12.91 & 0.58 & -0.93 & -0.24 & 0.33 \\
\hline 0.275 & 0.050 & 0.675 & 0.52 & 0.56 & 3.25 & 14.75 & 3.24 & 12.60 & 0.58 & -0.96 & -0.25 & 0.29 \\
\hline 0.300 & 0.050 & 0.650 & 0.55 & 0.59 & 3.14 & 14.11 & 3.12 & 11.09 & 0.53 & -1.10 & -0.28 & 0.13 \\
\hline 0.310 & 0.050 & 0.640 & 0.56 & 0.60 & 3.10 & 13.85 & 3.08 & 10.49 & 0.50 & -1.16 & -0.29 & 0.06 \\
\hline 0.320 & 0.050 & 0.630 & 0.57 & 0.61 & 3.06 & 13.60 & 3.03 & 9.90 & 0.48 & -1.21 & -0.30 & -0.02 \\
\hline 0.325 & 0.050 & 0.625 & 0.58 & 0.62 & 3.04 & 13.48 & 3.01 & 9.61 & 0.47 & -1.24 & -0.31 & -0.06 \\
\hline 0.340 & 0.050 & 0.610 & 0.59 & 0.63 & 2.99 & 13.11 & 2.94 & 8.75 & 0.44 & -1.33 & -0.33 & -0.18 \\
\hline 0.350 & 0.050 & 0.600 & 0.60 & 0.64 & 2.95 & 12.86 & 2.89 & 8.19 & 0.42 & -1.38 & -0.34 & -0.26 \\
\hline 0.360 & 0.050 & 0.590 & 0.61 & 0.65 & 2.91 & 12.62 & 2.85 & 7.65 & 0.39 & -1.43 & -0.35 & -0.35 \\
\hline 0.370 & 0.050 & 0.580 & 0.62 & 0.66 & 2.87 & 12.38 & 2.80 & 7.12 & 0.37 & -1.49 & -0.37 & -0.44 \\
\hline 0.380 & 0.050 & 0.570 & 0.63 & 0.66 & 2.83 & 12.13 & 2.76 & 6.62 & 0.35 & -1.54 & -0.38 & -0.53 \\
\hline 0.390 & 0.050 & 0.560 & 0.63 & 0.67 & 2.80 & 11.88 & 2.71 & 6.13 & 0.32 & -1.59 & -0.39 & -0.63 \\
\hline 0.400 & 0.050 & 0.550 & 0.64 & 0.68 & 2.76 & 11.62 & 2.67 & 5.66 & 0.30 & -1.63 & -0.41 & -0.72 \\
\hline 0.450 & 0.050 & 0.500 & 0.66 & 0.71 & 2.35 & 7.13 & 2.45 & 3.64 & 0.18 & -1.83 & -0.48 & -1.26 \\
\hline 0.500 & 0.050 & 0.450 & 0.67 & 0.73 & 2.11 & 5.51 & 2.24 & 2.18 & 0.06 & -1.92 & -0.56 & -1.86 \\
\hline 0.550 & 0.050 & 0.400 & 0.68 & 0.73 & 1.92 & 4.59 & 2.04 & 1.20 & -0.04 & -1.86 & -0.64 & -2.54 \\
\hline 0.600 & 0.050 & 0.350 & 0.67 & 0.72 & 1.77 & 3.93 & 1.86 & 0.61 & -0.10 & -1.61 & -0.72 & -3.28 \\
\hline 0.650 & 0.050 & 0.300 & 0.65 & 0.69 & 1.62 & 3.35 & 1.70 & 0.29 & -0.12 & -1.11 & -0.80 & -4.08 \\
\hline 0.700 & 0.050 & 0.250 & 0.61 & 0.64 & 1.46 & 2.81 & 1.57 & 0.12 & -0.06 & -0.28 & -0.88 & -4.95 \\
\hline 0.750 & 0.050 & 0.200 & 0.56 & 0.56 & 1.33 & 2.39 & 1.46 & 0.05 & 0.10 & 0.93 & -0.96 & -5.89 \\
\hline 0.800 & 0.050 & 0.150 & 0.49 & 0.46 & 1.21 & 2.03 & 1.39 & 0.02 & 0.38 & 2.60 & -1.04 & -6.88 \\
\hline 0.850 & 0.050 & 0.100 & 0.39 & 0.32 & 1.11 & 1.72 & 1.35 & 0.01 & 0.81 & 4.82 & -1.12 & -7.94 \\
\hline 0.870 & 0.050 & 0.080 & 0.34 & 0.26 & 1.07 & 1.61 & 1.35 & 0.00 & 1.03 & 5.87 & -1.15 & -8.38 \\
\hline 0.880 & 0.050 & 0.070 & 0.31 & 0.23 & 1.05 & 1.56 & 1.35 & 0.00 & 1.16 & 6.44 & -1.17 & -8.60 \\
\hline 0.890 & 0.050 & 0.060 & 0.28 & 0.19 & 1.03 & 1.51 & 1.35 & 0.00 & 1.29 & 7.04 & -1.18 & -8.82 \\
\hline 0.900 & 0.050 & 0.050 & 0.25 & 0.16 & 1.02 & 1.46 & 1.35 & 0.00 & 1.43 & 7.66 & -1.20 & -9.05 \\
\hline 0.910 & 0.050 & 0.040 & 0.20 & 0.12 & 1.01 & 1.41 & 1.36 & 0.00 & 1.58 & 8.31 & -1.21 & -9.28 \\
\hline 0.920 & 0.050 & 0.030 & 0.16 & 0.08 & 1.00 & 1.37 & 1.37 & 0.00 & 1.74 & 8.99 & -1.23 & -9.51 \\
\hline 0.930 & 0.050 & 0.020 & 0.11 & 0.04 & 1.00 & 1.33 & 1.38 & 0.00 & 1.90 & 9.70 & -1.24 & -9.75 \\
\hline 0.940 & 0.050 & 0.010 & 0.06 & 0 & 1.00 & 1.29 & 1.39 & 0.00 & 2.08 & 10.45 & -1.26 & -9.98 \\
\hline 0.950 & 0.050 & 0 & 0.01 & -0.05 & 1.00 & 1.27 & 1.41 & 0.00 & 2.27 & 11.22 & -1.27 & -10.22 \\
\hline
\end{tabular}


TABLE VII. Continuation of table (VI) for $y_{2}=0.075 \mathrm{~mol} \mathrm{~mol}^{-1}$.

\begin{tabular}{|c|c|c|c|c|c|c|c|c|c|c|c|c|}
\hline$y_{1}$ & $y_{2}$ & $y_{3}$ & $g_{s i m}^{e}$ & $g_{\text {mod }}^{e}$ & $\vartheta_{1, \text { sim }}$ & $\vartheta_{2, s i m}$ & $\vartheta_{1, \bmod }$ & $\vartheta_{2, \text { mod }}$ & $\Gamma_{11}$ & $\Gamma_{12}$ & $\Gamma_{21}$ & $\Gamma_{22}$ \\
\hline 0.025 & 0.075 & 0.900 & 0.28 & 0.29 & 4.35 & 21.64 & 4.53 & 21.98 & 0.94 & 0 & -0.03 & 0.76 \\
\hline 0.050 & 0.075 & 0.875 & 0.31 & 0.33 & 4.19 & 20.69 & 4.32 & 21.80 & 0.89 & -0.04 & -0.07 & 0.78 \\
\hline 0.070 & 0.075 & 0.855 & 0.34 & 0.36 & 4.08 & 19.97 & 4.16 & 21.41 & 0.85 & -0.10 & -0.11 & 0.78 \\
\hline 0.075 & 0.075 & 0.850 & 0.35 & 0.37 & 4.05 & 19.80 & 4.12 & 21.27 & 0.84 & -0.11 & -0.12 & 0.78 \\
\hline 0.100 & 0.075 & 0.825 & 0.38 & 0.41 & 3.92 & 18.94 & 3.95 & 20.41 & 0.80 & -0.22 & -0.17 & 0.75 \\
\hline 0.110 & 0.075 & 0.815 & 0.40 & 0.43 & 3.86 & 18.62 & 3.88 & 19.98 & 0.78 & -0.26 & -0.19 & 0.73 \\
\hline 0.120 & 0.075 & 0.805 & 0.41 & 0.44 & 3.81 & 18.29 & 3.81 & 19.50 & 0.76 & -0.32 & -0.21 & 0.70 \\
\hline 0.125 & 0.075 & 0.800 & 0.41 & 0.45 & 3.79 & 18.14 & 3.78 & 19.24 & 0.76 & -0.34 & -0.22 & 0.69 \\
\hline 0.130 & 0.075 & 0.795 & 0.42 & 0.46 & 3.76 & 17.98 & 3.75 & 18.97 & 0.75 & -0.37 & -0.24 & 0.67 \\
\hline 0.140 & 0.075 & 0.785 & 0.43 & 0.47 & 3.71 & 17.67 & 3.69 & 18.40 & 0.73 & -0.43 & -0.26 & 0.64 \\
\hline 0.150 & 0.075 & 0.775 & 0.45 & 0.49 & 3.66 & 17.36 & 3.62 & 17.80 & 0.71 & -0.50 & -0.28 & 0.60 \\
\hline 0.160 & 0.075 & 0.765 & 0.46 & 0.50 & 3.62 & 17.06 & 3.57 & 17.15 & 0.70 & -0.57 & -0.31 & 0.56 \\
\hline 0.170 & 0.075 & 0.755 & 0.47 & 0.52 & 3.57 & 16.77 & 3.51 & 16.48 & 0.68 & -0.64 & -0.33 & 0.51 \\
\hline 0.175 & 0.075 & 0.750 & 0.48 & 0.52 & 3.55 & 16.62 & 3.48 & 16.14 & 0.67 & -0.68 & -0.34 & 0.49 \\
\hline 0.180 & 0.075 & 0.745 & 0.48 & 0.53 & 3.52 & 16.48 & 3.45 & 15.78 & 0.67 & -0.71 & -0.35 & 0.46 \\
\hline 0.190 & 0.075 & 0.735 & 0.50 & 0.54 & 3.48 & 16.19 & 3.39 & 15.06 & 0.65 & -0.79 & -0.38 & 0.41 \\
\hline 0.200 & 0.075 & 0.725 & 0.51 & 0.56 & 3.43 & 15.91 & 3.34 & 14.33 & 0.63 & -0.87 & -0.40 & 0.35 \\
\hline 0.225 & 0.075 & 0.700 & 0.54 & 0.59 & 3.32 & 15.23 & 3.21 & 12.46 & 0.59 & -1.08 & -0.47 & 0.18 \\
\hline 0.250 & 0.075 & 0.675 & 0.56 & 0.61 & 3.22 & 14.56 & 3.08 & 10.59 & 0.55 & -1.30 & -0.54 & -0.01 \\
\hline 0.270 & 0.075 & 0.655 & 0.58 & 0.64 & 3.14 & 14.05 & 2.98 & 9.15 & 0.51 & -1.48 & -0.59 & -0.19 \\
\hline 0.275 & 0.075 & 0.650 & 0.59 & 0.64 & 3.12 & 13.92 & 2.96 & 8.80 & 0.51 & -1.52 & -0.61 & -0.23 \\
\hline 0.300 & 0.075 & 0.625 & 0.61 & 0.66 & 3.02 & 13.29 & 2.84 & 7.14 & 0.46 & -1.74 & -0.68 & -0.48 \\
\hline 0.310 & 0.075 & 0.615 & 0.62 & 0.67 & 2.98 & 13.04 & 2.80 & 6.52 & 0.44 & -1.83 & -0.71 & -0.58 \\
\hline 0.320 & 0.075 & 0.605 & 0.63 & 0.68 & 2.94 & 12.78 & 2.75 & 5.93 & 0.43 & -1.92 & -0.73 & -0.69 \\
\hline 0.325 & 0.075 & 0.600 & 0.64 & 0.68 & 2.92 & 12.66 & 2.73 & 5.65 & 0.42 & -1.96 & -0.75 & -0.75 \\
\hline 0.330 & 0.075 & 0.595 & 0.64 & 0.69 & 2.90 & 12.53 & 2.71 & 5.38 & 0.41 & -2.00 & -0.76 & -0.81 \\
\hline 0.340 & 0.075 & 0.585 & 0.65 & 0.70 & 2.86 & 12.21 & 2.66 & 4.85 & 0.39 & -2.09 & -0.79 & -0.92 \\
\hline 0.350 & 0.075 & 0.575 & 0.66 & 0.70 & 2.81 & 11.62 & 2.62 & 4.36 & 0.38 & -2.17 & -0.82 & -1.04 \\
\hline 0.360 & 0.075 & 0.565 & 0.66 & 0.71 & 2.74 & 10.87 & 2.58 & 3.91 & 0.36 & -2.24 & -0.85 & -1.17 \\
\hline 0.370 & 0.075 & 0.555 & 0.67 & 0.72 & 2.65 & 9.67 & 2.54 & 3.48 & 0.34 & -2.32 & -0.89 & -1.30 \\
\hline 0.375 & 0.075 & 0.550 & 0.67 & 0.72 & 2.59 & 8.93 & 2.51 & 3.28 & 0.33 & -2.35 & -0.90 & -1.36 \\
\hline 0.380 & 0.075 & 0.545 & 0.67 & 0.72 & 2.55 & 8.54 & 2.49 & 3.09 & 0.33 & -2.39 & -0.92 & -1.43 \\
\hline 0.390 & 0.075 & 0.535 & 0.67 & 0.73 & 2.47 & 7.71 & 2.45 & 2.74 & 0.31 & -2.46 & -0.95 & -1.57 \\
\hline 0.400 & 0.075 & 0.525 & 0.67 & 0.73 & 2.38 & 6.95 & 2.41 & 2.41 & 0.29 & -2.52 & -0.98 & -1.71 \\
\hline 0.425 & 0.075 & 0.500 & 0.68 & 0.74 & 2.25 & 6.06 & 2.32 & 1.72 & 0.26 & -2.65 & -1.06 & -2.08 \\
\hline 0.475 & 0.075 & 0.450 & 0.69 & 0.74 & 2.06 & 5.05 & 2.14 & 0.81 & 0.20 & -2.79 & -1.22 & -2.89 \\
\hline 0.575 & 0.075 & 0.350 & 0.68 & 0.70 & 1.74 & 3.73 & 1.84 & 0.13 & 0.21 & -2.36 & -1.55 & -4.79 \\
\hline 0.625 & 0.075 & 0.300 & 0.66 & 0.65 & 1.60 & 3.23 & 1.73 & 0.04 & 0.30 & -1.64 & -1.72 & -5.88 \\
\hline 0.675 & 0.075 & 0.250 & 0.63 & 0.58 & 1.45 & 2.73 & 1.65 & 0.01 & 0.48 & -0.47 & -1.89 & -7.05 \\
\hline 0.725 & 0.075 & 0.200 & 0.58 & 0.48 & 1.32 & 2.33 & 1.60 & 0.00 & 0.78 & 1.22 & -2.06 & -8.31 \\
\hline 0.775 & 0.075 & 0.150 & 0.50 & 0.34 & 1.21 & 1.99 & 1.60 & 0.00 & 1.21 & 3.52 & -2.22 & -9.66 \\
\hline 0.795 & 0.075 & 0.130 & 0.47 & 0.28 & 1.17 & 1.86 & 1.61 & 0.00 & 1.43 & 4.62 & -2.29 & -10.21 \\
\hline 0.825 & 0.075 & 0.100 & 0.40 & 0.18 & 1.10 & 1.68 & 1.64 & 0.00 & 1.82 & 6.55 & -2.38 & -11.08 \\
\hline 0.835 & 0.075 & 0.090 & 0.37 & 0.14 & 1.08 & 1.62 & 1.66 & 0.00 & 1.97 & 7.25 & -2.42 & -11.38 \\
\hline 0.845 & 0.075 & 0.080 & 0.35 & 0.10 & 1.07 & 1.58 & 1.68 & 0.00 & 2.12 & 7.98 & -2.45 & -11.67 \\
\hline 0.855 & 0.075 & 0.070 & 0.32 & 0.06 & 1.05 & 1.52 & 1.70 & 0.00 & 2.28 & 8.75 & -2.48 & -11.97 \\
\hline 0.865 & 0.075 & 0.060 & 0.29 & 0.01 & 1.03 & 1.48 & 1.73 & 0.00 & 2.46 & 9.55 & -2.51 & -12.28 \\
\hline 0.875 & 0.075 & 0.050 & 0.25 & -0.03 & 1.01 & 1.43 & 1.76 & 0.00 & 2.64 & 10.39 & -2.54 & -12.58 \\
\hline 0.905 & 0.075 & 0.020 & 0.12 & -0.17 & 1.00 & 1.31 & 1.87 & 0.00 & 3.24 & 13.14 & -2.63 & -13.52 \\
\hline 0.915 & 0.075 & 0.100 & 0.07 & -0.22 & 1.00 & 1.28 & 1.91 & 0.00 & 3.46 & 14.13 & -2.66 & -13.84 \\
\hline 0.925 & 0.075 & 0 & 0.02 & -0.28 & 1.00 & 1.25 & 1.97 & 0.00 & 3.69 & 15.16 & -2.69 & -14.16 \\
\hline
\end{tabular}


TABLE VIII. Spatially resolved vapor phase molar composition $b_{i 3}$ data. The normalized form $\left(b_{i 3}-b_{i 3, s}\right) / B_{i 3}$ can be computed with the following Spalding transfer numbers $B_{13}=-0.154$ and $B_{23}=-0.003$.

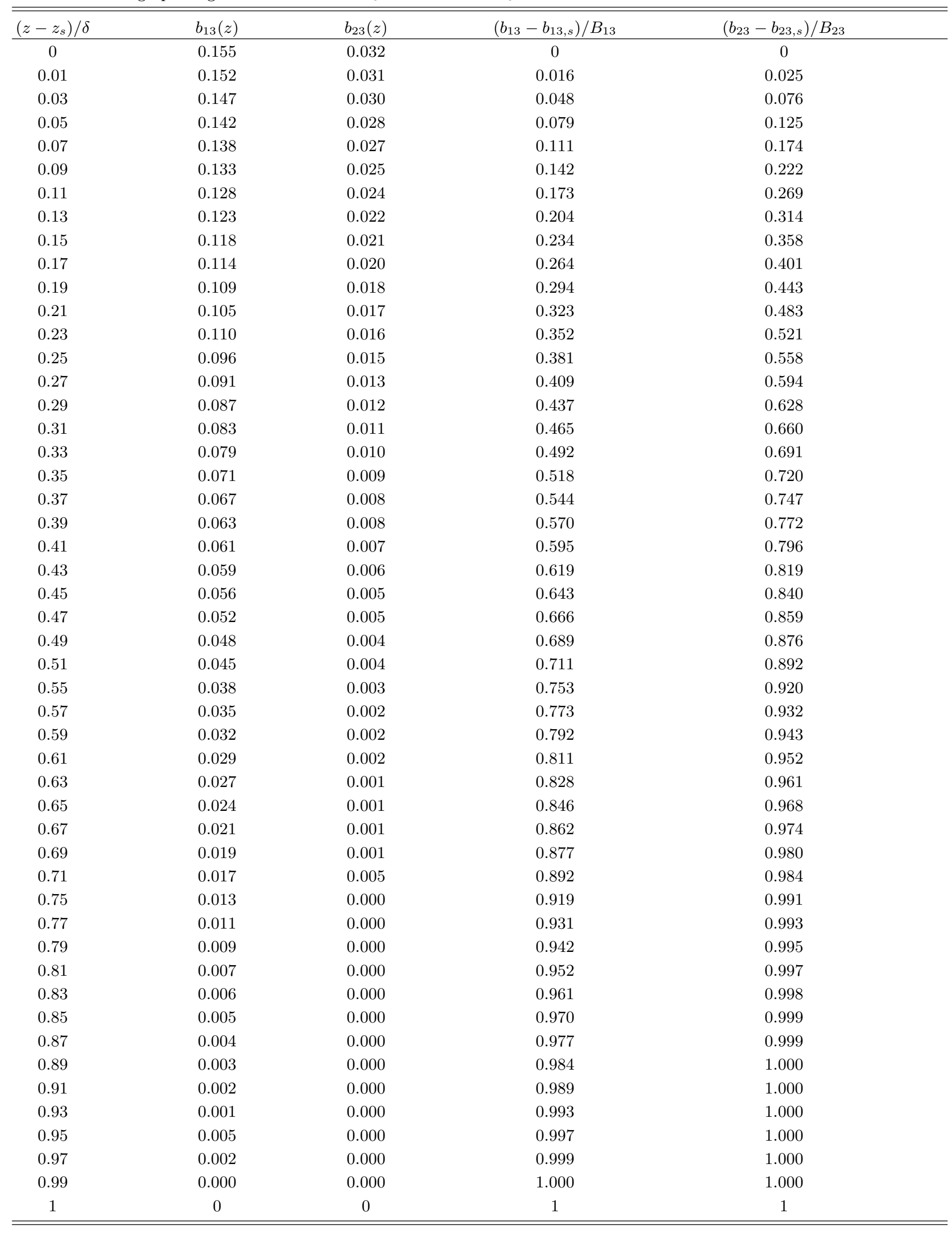




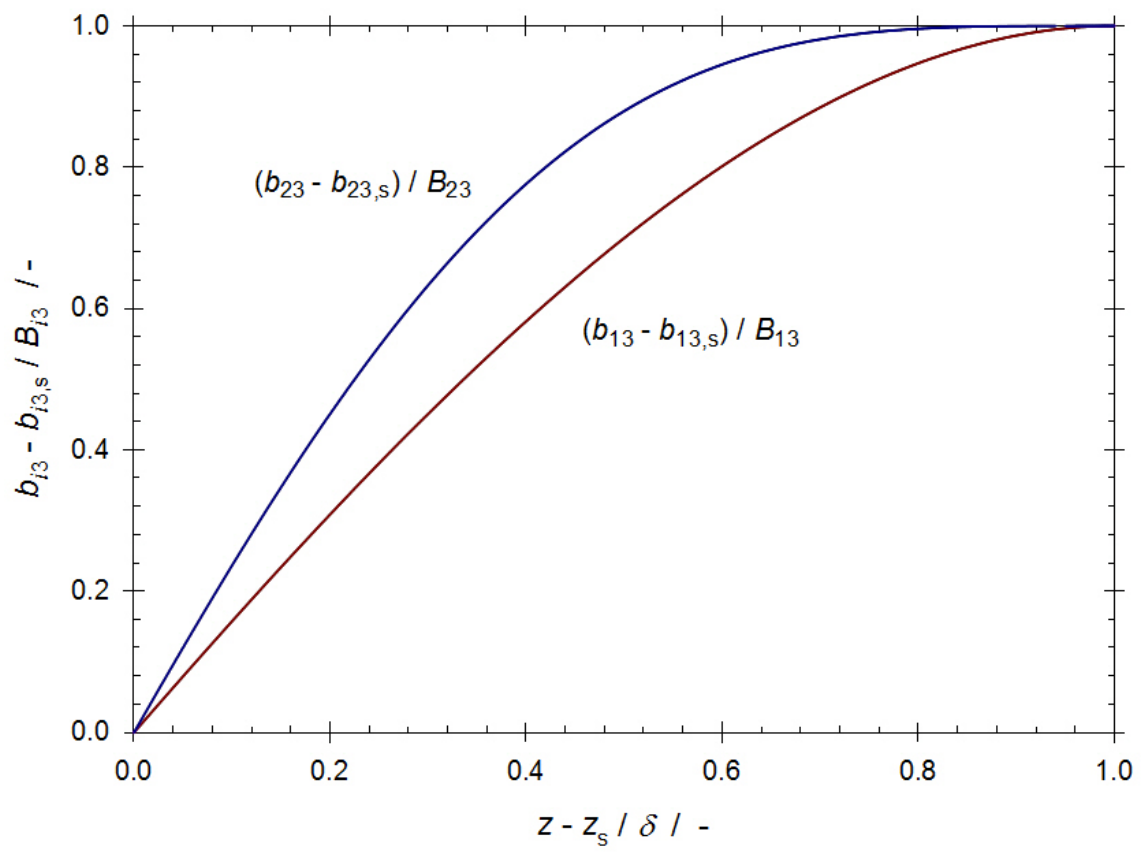

FIG. 5. The normalized vapor phase molar composition was assumed to be a classical boundary layer problem and hence can be considered analogous to the velocity distribution in a compressible boundary layer.

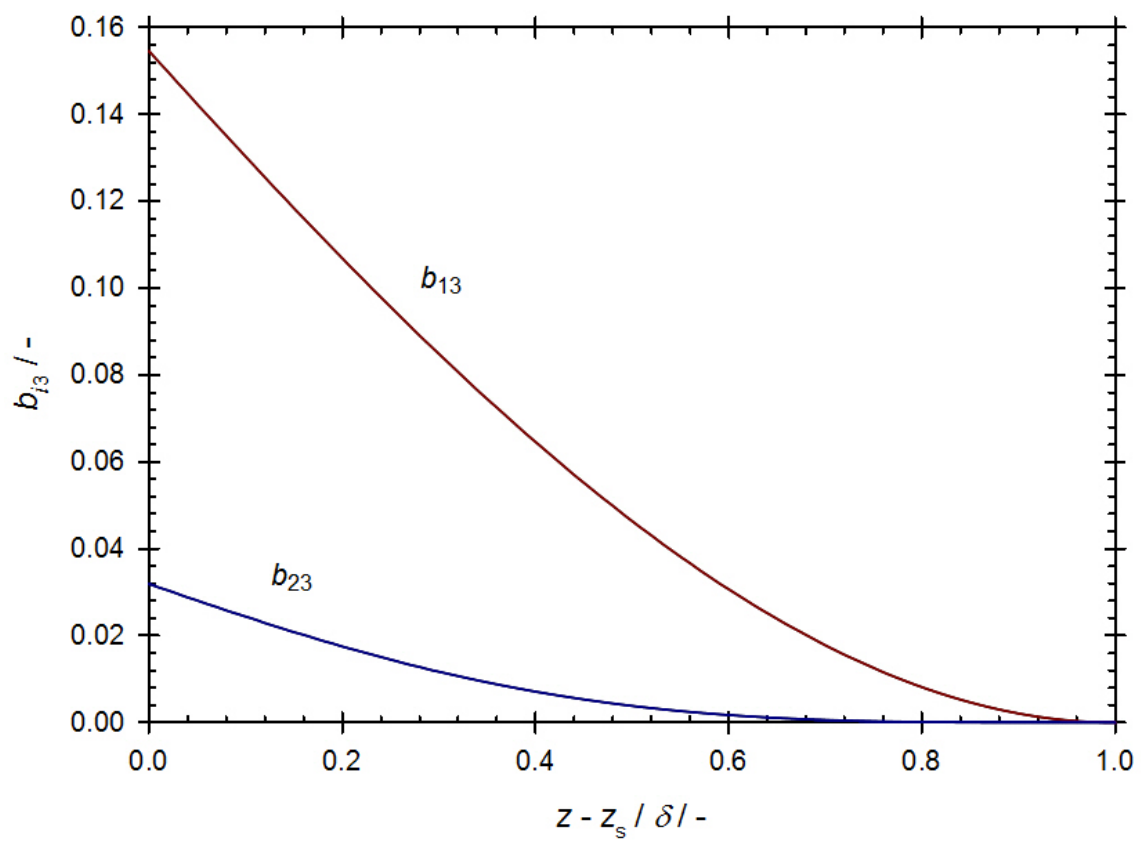

FIG. 6. Course of the weighted $i$-th component mole fraction $b_{i 3}(z)=y_{i}(z, t) / y_{3}(z, t)$ as a function of position. Both values approach $b_{i 3}=0$ for $z=z_{\delta}$ due to the enforced boundary condition. 
TABLE IX. Data for the liquid $\boldsymbol{x}$ and vapor $\boldsymbol{y}$ phase molar compositions, as well as the film thickness' time evolution. Since the disparity between the analytical solution and the NEMD simulations $\zeta_{\text {sim }}<\zeta_{\text {ana }}$ became substantial, disclosing further data after $t>213.50 \mathrm{~ns}$ was considered obsolete.

\begin{tabular}{|c|c|c|c|c|c|c|c|c|c|c|c|c|}
\hline$t / \mathrm{ns}$ & $x_{1, \text { sim }}$ & $x_{2, \text { sim }}$ & $x_{1, \text { ana }}$ & $x_{2, a n a}$ & $y_{1 s, s i m}$ & $y_{2 s, s i m}$ & $y_{3 s, s i m}$ & $y_{1 s, a n a}$ & $y_{2 s, a n a}$ & $y_{3 s, a n a}$ & $\zeta_{\text {sim }}$ & $\zeta_{\text {ana }}$ \\
\hline 0 & 0.495 & 0.505 & 0.495 & 0.505 & 0.130 & 0.027 & 0.843 & 0.130 & 0.027 & 0.843 & 1 & 1 \\
\hline 1.25 & 0.495 & 0.508 & 0.495 & 0.505 & 0.098 & 0.023 & 0.878 & 0.129 & 0.027 & 0.844 & 0.99 & 1.00 \\
\hline 3.25 & 0.495 & 0.517 & 0.485 & 0.515 & 0.078 & 0.021 & 0.901 & 0.127 & 0.027 & 0.846 & 0.97 & 0.97 \\
\hline 5.50 & 0.47 & 0.53 & 0.47 & 0.53 & 0.072 & 0.020 & 0.908 & 0.123 & 0.028 & 0.849 & 0.94 & 0.94 \\
\hline 7.50 & 0.46 & 0.54 & 0.46 & 0.54 & 0.070 & 0.020 & 0.910 & 0.120 & 0.029 & 0.851 & 0.92 & 0.92 \\
\hline 9.50 & 0.45 & 0.55 & 0.45 & 0.55 & 0.068 & 0.019 & 0.912 & 0.118 & 0.029 & 0.853 & 0.90 & 0.89 \\
\hline 11.50 & 0.44 & 0.56 & 0.44 & 0.56 & 0.067 & 0.020 & 0.913 & 0.115 & 0.030 & 0.855 & 0.88 & 0.87 \\
\hline 13.50 & 0.43 & 0.57 & 0.43 & 0.57 & 0.065 & 0.020 & 0.915 & 0.112 & 0.030 & 0.857 & 0.85 & 0.85 \\
\hline 15.50 & 0.42 & 0.58 & 0.42 & 0.58 & 0.063 & 0.021 & 0.916 & 0.110 & 0.031 & 0.860 & 0.83 & 0.83 \\
\hline 17.25 & 0.41 & 0.59 & 0.41 & 0.59 & 0.062 & 0.022 & 0.917 & 0.107 & 0.031 & 0.862 & 0.81 & 0.81 \\
\hline 19.25 & 0.49 & 0.60 & 0.40 & 0.60 & 0.059 & 0.022 & 0.919 & 0.105 & 0.032 & 0.864 & 0.79 & 0.80 \\
\hline 21.25 & 0.39 & 0.61 & 0.39 & 0.61 & 0.057 & 0.021 & 0.922 & 0.102 & 0.032 & 0.866 & 0.78 & 0.78 \\
\hline 23.00 & 0.38 & 0.62 & 0.38 & 0.62 & 0.056 & 0.021 & 0.923 & 0.099 & 0.033 & 0.868 & 0.76 & 0.76 \\
\hline 25.00 & 0.37 & 0.63 & 0.37 & 0.63 & 0.054 & 0.021 & 0.925 & 0.097 & 0.033 & 0.870 & 0.74 & 0.74 \\
\hline 26.75 & 0.36 & 0.64 & 0.36 & 0.64 & 0.052 & 0.021 & 0.927 & 0.094 & 0.034 & 0.872 & 0.73 & 0.73 \\
\hline 28.50 & 0.35 & 0.65 & 0.35 & 0.65 & 0.050 & 0.021 & 0.928 & 0.092 & 0.034 & 0.874 & 0.71 & 0.71 \\
\hline 30.50 & 0.34 & 0.66 & 0.34 & 0.66 & 0.049 & 0.022 & 0.929 & 0.089 & 0.035 & 0.876 & 0.69 & 0.70 \\
\hline 32.25 & 0.33 & 0.67 & 0.33 & 0.67 & 0.049 & 0.021 & 0.930 & 0.086 & 0.035 & 0.878 & 0.68 & 0.68 \\
\hline 34.00 & 0.32 & 0.68 & 0.32 & 0.68 & 0.047 & 0.021 & 0.931 & 0.084 & 0.036 & 0.880 & 0.66 & 0.67 \\
\hline 36.00 & 0.31 & 0.69 & 0.31 & 0.69 & 0.046 & 0.022 & 0.932 & 0.081 & 0.036 & 0.882 & 0.65 & 0.65 \\
\hline 37.75 & 0.30 & 0.70 & 0.30 & 0.70 & 0.044 & 0.023 & 0.933 & 0.078 & 0.037 & 0.885 & 0.63 & 0.64 \\
\hline 39.50 & 0.29 & 0.71 & 0.29 & 0.71 & 0.042 & 0.023 & 0.935 & 0.076 & 0.038 & 0.887 & 0.62 & 0.63 \\
\hline 41.50 & 0.28 & 0.72 & 0.28 & 0.72 & 0.041 & 0.023 & 0.936 & 0.073 & 0.038 & 0.889 & 0.61 & 0.61 \\
\hline 43.25 & 0.27 & 0.73 & 0.27 & 0.73 & 0.039 & 0.023 & 0.938 & 0.071 & 0.039 & 0.891 & 0.59 & 0.60 \\
\hline 45.25 & 0.26 & 0.74 & 0.26 & 0.74 & 0.037 & 0.024 & 0.939 & 0.068 & 0.039 & 0.893 & 0.58 & 0.59 \\
\hline 47.00 & 0.25 & 0.75 & 0.25 & 0.75 & 0.036 & 0.024 & 0.939 & 0.065 & 0.040 & 0.895 & 0.57 & 0.58 \\
\hline 49.00 & 0.23 & 0.77 & 0.24 & 0.76 & 0.035 & 0.024 & 0.941 & 0.063 & 0.040 & 0.897 & 0.56 & 0.57 \\
\hline 51.00 & 0.22 & 0.78 & 0.23 & 0.77 & 0.033 & 0.024 & 0.944 & 0.060 & 0.041 & 0.899 & 0.55 & 0.55 \\
\hline 53.00 & 0.21 & 0.79 & 0.22 & 0.78 & 0.031 & 0.023 & 0.946 & 0.058 & 0.041 & 0.901 & 0.53 & 0.54 \\
\hline 55.00 & 0.20 & 0.80 & 0.21 & 0.79 & 0.029 & 0.023 & 0.948 & 0.055 & 0.042 & 0.903 & 0.52 & 0.53 \\
\hline 57.00 & 0.19 & 0.81 & 0.20 & 0.80 & 0.028 & 0.024 & 0.948 & 0.052 & 0.042 & 0.905 & 0.51 & 0.52 \\
\hline 59.00 & 0.18 & 0.82 & 0.19 & 0.81 & 0.027 & 0.024 & 0.949 & 0.050 & 0.043 & 0.908 & 0.50 & 0.51 \\
\hline 61.25 & 0.17 & 0.83 & 0.18 & 0.82 & 0.025 & 0.024 & 0.951 & 0.047 & 0.043 & 0.910 & 0.49 & 0.50 \\
\hline 63.50 & 0.16 & 0.84 & 0.17 & 0.83 & 0.023 & 0.024 & 0.952 & 0.044 & 0.044 & 0.912 & 0.48 & 0.49 \\
\hline 65.75 & 0.15 & 0.85 & 0.16 & 0.84 & 0.022 & 0.025 & 0.953 & 0.042 & 0.044 & 0.914 & 0.47 & 0.48 \\
\hline 68.25 & 0.14 & 0.86 & 0.15 & 0.85 & 0.020 & 0.025 & 0.955 & 0.039 & 0.045 & 0.916 & 0.46 & 0.47 \\
\hline 70.75 & 0.13 & 0.87 & 0.14 & 0.86 & 0.019 & 0.025 & 0.956 & 0.037 & 0.045 & 0.918 & 0.45 & 0.46 \\
\hline 73.25 & 0.12 & 0.88 & 0.13 & 0.87 & 0.017 & 0.024 & 0.958 & 0.034 & 0.046 & 0.920 & 0.44 & 0.45 \\
\hline 76.00 & 0.11 & 0.89 & 0.12 & 0.88 & 0.016 & 0.025 & 0.959 & 0.031 & 0.047 & 0.922 & 0.43 & 0.44 \\
\hline 79.00 & 0.10 & 0.90 & 0.11 & 0.89 & 0.014 & 0.026 & 0.960 & 0.029 & 0.047 & 0.924 & 0.42 & 0.43 \\
\hline 82.00 & 0.09 & 0.91 & 0.10 & 0.90 & 0.013 & 0.025 & 0.962 & 0.026 & 0.048 & 0.926 & 0.41 & 0.42 \\
\hline 85.50 & 0.08 & 0.92 & 0.09 & 0.91 & 0.011 & 0.025 & 0.964 & 0.024 & 0.048 & 0.928 & 0.40 & 0.41 \\
\hline 89.25 & 0.07 & 0.93 & 0.08 & 0.92 & 0.010 & 0.026 & 0.964 & 0.021 & 0.049 & 0.930 & 0.39 & 0.40 \\
\hline 93.25 & 0.06 & 0.94 & 0.07 & 0.93 & 0.008 & 0.025 & 0.966 & 0.018 & 0.049 & 0.933 & 0.38 & 0.39 \\
\hline 98.00 & 0.05 & 0.95 & 0.06 & 0.94 & 0.007 & 0.026 & 0.967 & 0.016 & 0.050 & 0.935 & 0.37 & 0.38 \\
\hline 103.25 & 0.04 & 0.96 & 0.05 & 0.95 & 0.005 & 0.027 & 0.968 & 0.013 & 0.050 & 0.937 & 0.35 & 0.36 \\
\hline 109.75 & 0.03 & 0.97 & 0.04 & 0.96 & 0.004 & 0.025 & 0.972 & 0.010 & 0.051 & 0.939 & 0.34 & 0.35 \\
\hline 117.75 & 0.02 & 0.98 & 0.03 & 0.97 & 0.003 & 0.027 & 0.970 & 0.008 & 0.051 & 0.941 & 0.33 & 0.34 \\
\hline 128.75 & 0.01 & 0.99 & 0.02 & 0.98 & 0.002 & 0.028 & 0.971 & 0.005 & 0.052 & 0.943 & 0.31 & 0.32 \\
\hline 147.25 & 0.01 & 0.99 & 0.01 & 0.99 & 0.001 & 0.027 & 0.972 & 0.003 & 0.052 & 0.945 & 0.28 & 0.29 \\
\hline 150.25 & 0.006 & 0.994 & 0.009 & 0.991 & 0.001 & 0.027 & 0.972 & 0.002 & 0.052 & 0.945 & 0.27 & 0.29 \\
\hline
\end{tabular}




\begin{tabular}{ccccccccccccc}
\hline \hline$t / \mathrm{ns}$ & $x_{1, \text { sim }}$ & $x_{2, \text { sim }}$ & $x_{1, \text { ana }}$ & $x_{2, \text { ana }}$ & $y_{1, \text { sim }}$ & $y_{2, \text { sim }}$ & $y_{3, \text { sim }}$ & $y_{1, \text { ana }}$ & $y_{2, \text { ana }}$ & $y_{3, \text { ana }}$ & $\zeta_{\text {sim }}$ & $\zeta_{\text {ana }}$ \\
\hline 153.25 & 0.006 & 0.994 & 0.008 & 0.992 & 0.000 & 0.027 & 0.973 & 0.002 & 0.052 & 0.945 & 0.27 & 0.29 \\
156.75 & 0.005 & 0.995 & 0.007 & 0.993 & 0.000 & 0.027 & 0.973 & 0.002 & 0.052 & 0.946 & 0.26 & 0.28 \\
165.75 & 0.004 & 0.996 & 0.005 & 0.995 & 0.000 & 0.027 & 0.973 & 0.001 & 0.053 & 0.946 & 0.25 & 0.27 \\
171.50 & 0.005 & 0.995 & 0.004 & 0.996 & 0.000 & 0.027 & 0.973 & 0.001 & 0.053 & 0.946 & 0.24 & 0.26 \\
189.50 & 0.004 & 0.996 & 0.002 & 0.998 & 0.000 & 0.027 & 0.973 & 0.001 & 0.053 & 0.947 & 0.22 & 0.25 \\
207.75 & 0.003 & 0.997 & 0.001 & 0.999 & 0.000 & 0.027 & 0.973 & 0.000 & 0.053 & 0.947 & 0.189 & 0.229 \\
210.50 & 0.0038 & 0.996 & 0.0009 & 0.9991 & 0.000 & 0.028 & 0.972 & 0.000 & 0.053 & 0.947 & 0.185 & 0.226 \\
213.50 & 0.0032 & 0.997 & 0.0008 & 0.9992 & 0.000 & 0.026 & 0.974 & 0.000 & 0.053 & 0.947 & 0.181 & 0.223 \\
\hline \hline
\end{tabular}

TABLE X. Comparing the deviation between the analytical solution liquid density time evolution to the NEMD simulation's, justifies the assumption that has been made in (33).

\begin{tabular}{|c|c|c|c|}
\hline$t / \mathrm{ns}$ & $\rho_{l, a n a}$ & $\rho_{l, s i m}$ & dev. in $\%$ \\
\hline 0.31 & 46.097 & 46.642 & 1.17 \\
\hline 2.56 & 46.172 & 46.694 & 1.12 \\
\hline 4.77 & 46.246 & 46.757 & 1.09 \\
\hline 9.07 & 46.395 & 46.903 & 1.08 \\
\hline 21.33 & 46.842 & 47.348 & 1.07 \\
\hline 25.27 & 46.991 & 47.495 & 1.06 \\
\hline 29.16 & 47.140 & 47.643 & 1.06 \\
\hline 38.86 & 47.513 & 48.018 & 1.05 \\
\hline 48.76 & 47.885 & 48.375 & 1.01 \\
\hline 50.81 & 47.960 & 48.448 & 1.01 \\
\hline 54.99 & 48.109 & 48.577 & 0.96 \\
\hline 57.14 & 48.183 & 48.663 & 0.99 \\
\hline 59.34 & 48.258 & 48.719 & 0.95 \\
\hline 61.60 & 48.332 & 48.776 & 0.91 \\
\hline 63.91 & 48.407 & 48.859 & 0.92 \\
\hline 66.30 & 48.481 & 48.913 & 0.88 \\
\hline 68.77 & 48.556 & 48.974 & 0.85 \\
\hline 71.34 & 48.630 & 49.053 & 0.86 \\
\hline 74.02 & 48.705 & 49.104 & 0.81 \\
\hline 86.31 & 49.003 & 49.346 & 0.70 \\
\hline 89.95 & 49.078 & 49.407 & 0.67 \\
\hline 93.93 & 49.152 & 49.448 & 0.60 \\
\hline 103.33 & 49.301 & 49.561 & 0.52 \\
\hline 109.09 & 49.376 & 49.610 & 0.47 \\
\hline 116.00 & 49.450 & 49.636 & 0.37 \\
\hline 124.71 & 49.525 & 49.672 & 0.30 \\
\hline 136.73 & 49.599 & 49.699 & 0.20 \\
\hline 156.85 & 49.674 & 49.723 & 0.10 \\
\hline 163.25 & 49.689 & 49.730 & 0.08 \\
\hline 171.47 & 49.703 & 49.720 & 0.03 \\
\hline 176.67 & 49.711 & 49.718 & 0.01 \\
\hline 183.01 & 49.718 & 49.741 & 0.04 \\
\hline 191.17 & 49.726 & 49.740 & 0.03 \\
\hline 202.65 & 49.733 & 49.740 & 0.01 \\
\hline
\end{tabular}

